\title{
Kadomtsev-Petviashvili turning points and CKP hierarchy
}

\author{
I. Krichever* $\quad$ A. Zabrodin ${ }^{\dagger}$
}

December 2020

\begin{abstract}
A characterization of the Kadomtsev-Petviashvili hierarchy of type $\mathrm{C}(\mathrm{CKP})$ in terms of the KP tau-function is given. Namely, we prove that the CKP hierarchy can be identified with the restriction of odd times flows of the KP hierarchy on the locus of turning points of the second flow. The notion of CKP tau-function is clarified and connected with the KP tau function. Algebraic-geometrical solutions and in particular elliptic solutions are discussed in detail. The new identity for theta-functions of curves with holomorphic involution having fixed points is obtained.
\end{abstract}

\section{Contents}

1 Introduction $\quad 2$

2 The CKP hierarchy 5

2.1 The $\mathcal{L}$-operator and the dressing operator . . . . . . . . . . . . . 5 5

2.2 The wave function and the tau-function . . . . . . . . . . 7

2.3 CKP hierarchy versus KP hierarchy . . . . . . . . . . . . . 11

*Skolkovo Institute of Science and Technology, 143026, Moscow, Russia and National Research University Higher School of Economics, 20 Myasnitskaya Ulitsa, Moscow 101000, Russia, and Columbia University, New York, USA; e-mail: krichev@math.columbia.edu

${ }^{\dagger}$ National Research University Higher School of Economics, 20 Myasnitskaya Ulitsa, Moscow 101000, Russia and Skolkovo Institute of Science and Technology, 143026, Moscow, Russia and ITEP NRC KI, 25 B.Cheremushkinskaya, Moscow 117218, Russia; e-mail: zabrodin@itep.ru 
3 Algebraic-geometrical solutions to the KP and CKP hierarchies

3.1 Prelimineries . . . . . . . . . . . . . . . . . . . . . . . 16

3.2 The Baker-Akhiezer function . . . . . . . . . . . . . . . . . 19

3.3 The dual Baker-Akhiezer function . . . . . . . . . . . . . . . . . 20

3.4 Theta-functional formulae . . . . . . . . . . . . . 22

3.5 The tau-function . . . . . . . . . . . . . . . . 23

3.6 Curves with involution: solutions to the CKP hierarchy . . . . . . . . 24

3.7 Degeneration of algebraic-geometrical solutions: soliton solutions . . . . 28

4 Elliptic solutions $\quad 30$

4.1 The generating problem . . . . . . . . . . . . . . . 31

4.2 The integrals of motion and the spectral curve . . . . . . . 34

4.3 The wave function as the Baker-Akhiezer function on the spectral curve . 35

4.4 Degenerations of elliptic solutions . . . . . . . . . . . . . . 36

4.4 .1 Trigonometric solutions . . . . . . . . . . . . . . 36

4.4 .2 Rational solutions . . . . . . . . . . . . . . . . . 37

5 Concluding remarks

Acknowledgments

$\begin{array}{ll}\text { Appendix A } & 39\end{array}$

Appendix B

References 41

\section{Introduction}

The Kadomtsev-Petviashvily (KP) hierarchy is one of the most fundamental in the modern theory of integrable systems. It has at least three well-known definitions/representations. In its original, the so-called Zakharov-Shabat form [1], it is an infinite system of equations on an infinite number of variables which are the coefficients of monic ordinary linear differential operators

$$
B_{k}=\partial_{x}^{k}+\sum_{i=0}^{k-2} u_{k, i}(x, \mathbf{t}) \partial_{x}^{i}
$$


depending on $x$ and an infinite set of "times" $\mathbf{t}=\left\{t_{1}, t_{2}, t_{3}, \ldots\right\}$. The equations of the hierarchy are equivalent to the operator equations

$$
\partial_{t_{l}} B_{k}-\partial_{t_{k}} B_{l}+\left[B_{k}, B_{l}\right]=0, \quad \text { for all pairs } k, l .
$$

For each pair $(k, l)$ the operator equation (1.2) is equivalent to a system of partial differential equations on the coefficients of the operators $B_{k}, B_{l}$. The system is well-defined in the sense that the number of equations is equal to the number of unknown functions. For example, for the case $k=2, l=3$ in which $B_{2}=\partial_{x}^{2}+2 u$ and $B_{3}=\partial_{x}^{3}+3 u \partial_{x}+w$ equation (1.2) is equivalent to a system of two equations for $u$ and $w$. After eliminating $w$ from this system, and after the change of the notation for independent variables $t_{2}=y, t_{3}=t$, the remaining equation for $u$ becomes the original KP equation

$$
3 u_{y y}=\left(4 u_{t}-12 u u_{x}-u_{x x x}\right)_{x} .
$$

Remark. Under the assumption that $u$ is a periodic function of the variables $x, y$, i.e. $u\left(x+\ell_{1}, y\right)=u\left(x, y+\ell_{2}\right)=u(x, y)$, the KP hierarchy can be defined as a set of commuting flows on the space of Cauchy data for (1.3): the space of one periodic function of two variables [2].

The second form of the KP hierarchy (which is often called the Sato form) was introduced in [3] as a system of commuting flows on the space of sequences $\left(u_{1}(x), u_{2}(x), \ldots\right)$ of functions of one variable $x$, which can be identified with the space of pseudo-differential operators of the form

$$
\mathcal{L}=\partial_{x}+u_{1} \partial_{x}^{-1}+u_{2} \partial_{x}^{-2}+\ldots
$$

The flows are defined by the Lax equations

$$
\partial_{t_{k}} \mathcal{L}=\left[B_{k}, \mathcal{L}\right], \quad B_{k}=\left(\mathcal{L}^{k}\right)_{+}, \quad k=1,2,3, \ldots
$$

where $(\cdot)_{+}$stands for the differential part of a pseudo-differential operator. The statement that equations (1.2) follow from equations (1.5) is easy. The inverse statement is true up to a triangular change of time variables [4].

The third form of the KP hierarchy is an infinite system of equations for one function $\tau^{\mathrm{KP}}(\mathbf{t})$ of an infinite number of variables generated by the Hirota bilinear equation [5, 6]

$$
\oint_{C_{\infty}} \tau^{\mathrm{KP}}\left(\mathbf{t}-\left[z^{-1}\right]\right) \tau^{\mathrm{KP}}\left(\mathbf{t}^{\prime}+\left[z^{-1}\right]\right) \exp \left(\sum_{k \geq 1}\left(t_{k}-t_{k}^{\prime}\right) z^{k}\right) d z=0,
$$

which should be valid for all sets of times $\mathbf{t}, \mathbf{t}^{\prime}$. Here and below $C_{\infty}$ is a big circle around the infinity $z=\infty$ and $\mathbf{t} \pm\left[z^{-1}\right]$ denotes the following special shift of time variables:

$$
\mathbf{t} \pm\left[z^{-1}\right]:=\left\{t_{1} \pm \frac{1}{z}, t_{2} \pm \frac{1}{2 z^{2}}, t_{3} \pm \frac{1}{3 z^{3}}, \ldots\right\}
$$

Note that equation (1.6) is invariant under the transformation

$$
\tau^{\mathrm{KP}}(x, \mathbf{t}) \longrightarrow \exp \left(\gamma_{0}+\gamma_{1} x+\sum_{j \geq 1} \gamma_{j} t_{j}\right) \tau^{\mathrm{KP}}(x, \mathbf{t})
$$


with constant $\gamma_{j}$. The transformed tau-function corresponds to the same solution of the KP equations in the Sato or Zakharov-Shabat form. The tau-functions which differ by multiplication by exponent of a linear function of times are called equivalent.

In [7] an infinite integrable hierarchy of partial differential equations with $\operatorname{Sp}(\infty)$ symmetry was introduced and called the Kadomtsev-Petviashvili hierarchy of type C (CKP). It is a hierarchy of commuting flows that are the restriction of the flows of the KP hierarchy corresponding to "odd" times $\mathbf{t}_{\mathrm{o}}=\left\{t_{1}, t_{3}, t_{5}, \ldots\right\}$ onto the space of anti self-adjoint pseudo-differential operators $\mathcal{L}_{\text {CKP }}$ of the form (1.4), i.e. such that

$$
\mathcal{L}_{\mathrm{CKP}}^{\dagger}=-\mathcal{L}_{\mathrm{CKP}}
$$

where $\dagger$ means the formal adjoint defined by the rule $\left(f(x) \circ \partial_{x}^{m}\right)^{\dagger}=\left(-\partial_{x}\right)^{m} \circ f(x)$. The CKP hierarchy was revisited in [8, 9, 10, 11].

The first goal of this work is to characterize the CKP hierarchy in terms of the KP tau-function. More precisely, each solution of the CKP hierarchy has a unique extension to the solution of the full KP hierachy via the flows (1.5) with even $k$ (which obviously do not preserve constraint (1.9) ). In what follows we will refer to the corresponding solution to the KP hierarchy as KP extension of the solution to the CKP hierarchy. In section 2 we prove that the KP tau-function is the tau-function of such a solution if the equation

$$
\left.\partial_{t_{2}} \log \tau^{\mathrm{KP}}\right|_{\mathbf{t}_{\mathrm{e}}=0}=0
$$

holds for all $\mathbf{t}_{\mathrm{o}}$, where all "even" times $\mathbf{t}_{\mathrm{e}}=\left(t_{2}, t_{4}, \ldots\right)$ are set equal to zero. Conversely, in the equivalence class of any KP tau-function which is the tau-function of the KP extension of a solution to the CKP hierarchy there exists one which satisfies the condition (1.10). We note that this condition selects "turning points" of the KP hierarchy in the sense that if $x_{i}$ are zeros of the tau-function $\tau^{\mathrm{KP}}(x, \mathbf{t})$, i.e., $\tau^{\mathrm{KP}}\left(x_{i}, \mathbf{t}\right)=0$, then $\partial_{t_{2}} x_{i}\left(t_{1}, t_{3}, \ldots\right)=0$ for all $t_{1}, t_{3}, t_{5}, \ldots$.

We also prove the existence of the tau-function $\tau\left(x, \mathbf{t}_{\mathrm{o}}\right)$ for the CKP hierarchy which is a function of "odd" times $\mathbf{t}_{\mathrm{o}}$ only and prove that it is the square root of $\tau^{\mathrm{KP}}$ satisfying the condition (1.10):

$$
\tau\left(t_{1}, t_{3}, t_{5}, \ldots,\right)=\sqrt{\tau^{\mathrm{KP}}\left(t_{1}, 0, t_{3}, 0, t_{5}, 0, \ldots\right)}
$$

In the first part of Section 3 we present in detail the algebraic-geometrical construction of quasi-periodic solutions to the CKP hierarchy briefly outlined in [7]. We start from the general scheme proposed in [12, 13]. The specialization for the CKP hierarchy is a certain reduction of this general scheme. The data defining the algebraic-geometrical solutions of the CKP hierarchy are the following: a smooth algebraic curve $\Gamma$ of genus $g$ with a holomorphic involution having at least one fixed point $P_{\infty} \in \Gamma$, a local parameter in a neighborhood of $P_{\infty}$ which is odd with respect to the involution and a generic admissible divisor of degree $g$. The locus of the admissible divisors in the Jacobian is a translate of the Prym variety of $\Gamma$. In the second part of Section 3 we prove the new identity for the Riemann theta-function of a curve with involution having at least one fixed point (Theorem 3.4). The identity is an algebraic-geometrical incarnation of the relations between KP- and CKP-tau-functions discussed in Section 2. 
In Section 4 we study double-periodic (elliptic) in the variable $x=t_{1}$ solutions to the $C$-version of the KP equation and their trigonometric and rational degenerations.

In the seminal paper [14] the motion of poles of singular solutions to the Korteweg-de Vries and Boussinesq equations was considered. It was discovered that the poles move as particles of the many-body Calogero-Moser system [15, 16, 17] with some additional restrictions in the phase space. In [18, 19] it was shown that in the case of the KP equation this correspondence becomes an isomorphism: the dynamics of poles of rational solutions to the KP equation is given by equations of motion for the Calogero-Moser system with pairwise interaction potential $1 /\left(x_{i}-x_{j}\right)^{2}$. This remarkable connection was further generalized to elliptic solutions in [20]: poles $x_{i}$ of the elliptic solutions as functions of $t_{2}=y$ move according to the equations of motion

$$
\partial_{y}^{2} x_{i}=4 \sum_{k \neq i} \wp^{\prime}\left(x_{i}-x_{k}\right)
$$

of Calogero-Moser particles with the elliptic interaction potential $\wp\left(x_{i}-x_{j}\right)$ ( $\wp$ is the Weierstrass $\wp$-function). Moreover, in [20] it was shown that the origin of equations (1.12) is related to a more fundamental problem: when a linear equation with elliptic coefficients has double-Bloch solutions (i.e. solution which are sections of a line bundle over the elliptic curve, see [21]). Recently, the method proposed in [20] was applied to the theory of elliptic solutions of the BKP equation [22, 23].

Along the same line of arguments we derive in Section 4 the equations of motion for poles of elliptic solutions to the CKP equation:

$$
\dot{x}_{i}=3 \sum_{k \neq i}^{n} \wp\left(x_{i}-x_{k}\right)-6 c,
$$

where $c$ is a constant and dot means the $t_{3}$-derivative. In contrast to the KP and BKP cases, where the equations of motion are of the second order (see [20, 22, 23]) equations (1.13) are of the first order. As follows from the comparison of the CKP and KP hierarchies in Section 2 equation (1.13) coincide with the restriction of the Calogero-Moser flow corresponding to the higher Hamiltonian $H_{3}$ to the manifold of turning points in the $2 n$-dimensional phase space $\left(p_{i}, x_{i}\right)$, i.e. the $n$-dimensional submanifold $p_{i}=\partial_{y} x_{i}=0$ for all $i=1, \ldots, n$.

Remark. The notion of the turning points of the elliptic Calogero-Moser system and the study of the corresponding spectral curves in the forthcoming paper [24] was motivated by the problem of construction of explicit solutions to the two-dimensional $O(2 m+1)$ sigma-model.

\section{The CKP hierarchy}

\subsection{The $\mathcal{L}$-operator and the dressing operator}

The set of independent variables ("times") in the CKP hierarchy is $\mathbf{t}_{\mathrm{o}}=\left\{t_{1}, t_{3}, t_{5}, \ldots\right\}$. Like in the BKP case, they are indexed by positive odd numbers. It is convenient to set 
$t_{1}=x+$ const, so that the vector fields $\partial_{t_{1}}$ and $\partial_{x}$ are identical: $\partial_{t_{1}}=\partial_{x}$. The hierarchy is defined on the space of pseudo-differential operators $\mathcal{L}_{\mathrm{CKP}}$ of the form

$$
\mathcal{L}_{\mathrm{CKP}}=\partial_{x}+u_{1} \partial_{x}^{-1}+u_{2} \partial_{x}^{-2}+\ldots
$$

subject to the constraint

$$
\mathcal{L}_{\mathrm{CKP}}^{\dagger}=-\mathcal{L}_{\mathrm{CKP}},
$$

The coefficients $u_{j}$ of $\mathcal{L}_{\mathrm{CKP}}$ depend on $x$ and on all the times. It is convenient to introduce the wave operator (or dressing operator)

$$
W=1+\xi_{1} \partial_{x}^{-1}+\xi_{2} \partial_{x}^{-2}+\ldots
$$

such that

$$
\mathcal{L}_{\mathrm{CKP}}=W \partial_{x} W^{-1} .
$$

The wave operator is unique up to multiplication from the right by a pseudo-differential operator with constant coefficients.

The constraint (2.2) implies that $W^{\dagger} W$ commutes with $\partial_{x}$, i.e., it is a pseudodifferential operator with constant coefficients. We fix the above mentioned ambiguity in the definition of the wave operator by imposing the equation $W^{\dagger} W=1$, i.e.

$$
W^{\dagger}=W^{-1} \text {. }
$$

The hierachy of flows is defined by the Lax equations

$$
\partial_{t_{k}} \mathcal{L}_{\mathrm{CKP}}=\left[B_{k}, \mathcal{L}_{\mathrm{CKP}}\right], \quad B_{k}=\left(\mathcal{L}_{\mathrm{CKP}}^{k}\right)_{+}, \quad k=1,3,5, \ldots,
$$

which obviously preserve the constraint (2.5) since $B_{k}^{\dagger}=-B_{k}$ (for odd $k$ ).

The zero curvature (Zakharov-Shabat) equations

$$
\partial_{t_{l}} B_{k}-\partial_{t_{k}} B_{l}+\left[B_{k}, B_{l}\right]=0, \quad k, l \text { odd }
$$

is an easy corollary of (2.6). They are equivalent to the statement that the flows (2.6) commute with each other.

The first equation of the CKP hierarchy is the equation $\partial_{t_{3}} B_{5}-\partial_{t_{5}} B_{3}+\left[B_{5}, B_{3}\right]=0$ with

$$
\begin{gathered}
B_{3}=\partial_{x}^{3}+6 u \partial_{x}+3 u^{\prime}, \quad u^{\prime} \equiv \partial_{x} u, \quad u=\frac{1}{2} u_{1}, \\
B_{5}=\partial_{x}^{5}+10 u \partial_{x}^{3}+15 u^{\prime} \partial_{x}^{2}+v \partial_{x}+\frac{1}{2}\left(v^{\prime}-5 u^{\prime \prime \prime}\right) .
\end{gathered}
$$

Straightforward calculations give the following system of equations for the unknown functions $u, v$ :

$$
\left\{\begin{array}{l}
10 \partial_{t_{3}} u=3 v^{\prime}-35 u^{\prime \prime \prime}-120 u u^{\prime} \\
6 \partial_{t_{5}} u-\partial_{t_{3}} v=\frac{57}{2} u^{\prime \prime \prime \prime \prime}+150 u u^{\prime \prime \prime}+180 u^{\prime} u^{\prime \prime}-\frac{5}{2} v^{\prime \prime \prime}+6 v u^{\prime}-6 u v^{\prime}
\end{array}\right.
$$

Note that the variable $v$ can be excluded by passing to the unknown function $U$ such that $U^{\prime}=u$. 


\subsection{The wave function and the tau-function}

The Lax equations (2.6) are compatibility conditions of the auxiliary linear problems

$$
\partial_{t_{k}} \Psi=B_{k} \Psi, \quad \mathcal{L}_{\mathrm{CKP}} \Psi=z \Psi
$$

for the formal wave function

$$
\Psi=\Psi\left(\mathbf{t}_{\mathrm{o}}, z\right)=W e^{x z+\zeta\left(\mathbf{t}_{\mathrm{o}}, z\right)}
$$

where $z$ is the spectral parameter and

$$
\zeta\left(\mathbf{t}_{\mathrm{o}}, z\right)=\sum_{k \geq 1, \text { odd }} t_{k} z^{k}
$$

Note that the operator $\partial_{x}^{-1}$ acts to the exponential function as $\partial_{x}^{-1} e^{x z}=z^{-1} e^{x z}$. Therefore, from (2.3),$(2.12)$, it follows that the wave function has the following expansion as $z \rightarrow \infty$ :

$$
\Psi\left(x, \mathbf{t}_{\mathrm{o}}, z\right)=e^{x z+\zeta\left(\mathbf{t}_{\mathrm{o}}, z\right)}\left(1+\sum_{k \geq 1} \xi_{k} z^{-k}\right) .
$$

Proposition 2.1 ([7]) The wave function $\Psi$ satisfies the bilinear relation

$$
\oint_{C_{\infty}} \Psi\left(x, \mathbf{t}_{\mathrm{o}}, z\right) \Psi\left(x, \mathbf{t}_{\mathrm{o}}^{\prime},-z\right) d z=0
$$

for all $\mathbf{t}_{\mathrm{o}}, \mathbf{t}_{\mathrm{o}}^{\prime}$.

For completeness, we give a sketch of the proof here. By virtue of differential equations (2.6), the bilinear relation is equivalent to vanishing of the coefficients

$$
b_{m}=\left.\frac{1}{2 \pi i} \partial_{x^{\prime}}^{m} \oint_{C_{\infty}} \Psi\left(x, \mathbf{t}_{\mathrm{o}}, z\right) \Psi\left(x^{\prime}, \mathbf{t}_{\mathrm{o}},-z\right) d z\right|_{x^{\prime}=x} \quad \text { for all } m \geq 0 .
$$

We have:

$$
\begin{gathered}
b_{m}=\left.\frac{1}{2 \pi i} \oint_{C_{\infty}}\left(\sum_{k \geq 0} \xi_{k}(x) z^{-k}\right) \partial_{x^{\prime}}^{m}\left(\sum_{l \geq 0} \xi_{l}\left(x^{\prime}\right)(-z)^{-l}\right) e^{\left(x-x^{\prime}\right) z} d z\right|_{x^{\prime}=x} \\
=\frac{1}{2 \pi i} \oint_{C_{\infty}}\left(\sum_{k \geq 0} \xi_{k} z^{-k}\right)\left(\partial_{x}-z\right)^{m}\left(\sum_{l \geq 0} \xi_{l}(-z)^{-l}\right) d z \\
=\sum_{j+k+l=m+1}(-1)^{m+j+l}\left(\begin{array}{c}
m \\
j
\end{array}\right) \xi_{k} \partial_{x}^{j} \xi_{l} .
\end{gathered}
$$

The last expression is the coefficient at $(-1)^{m} \partial_{x}^{-m-1}$ in the operator $W W^{\dagger}$ :

$$
W W^{\dagger}=1+\sum_{m \geq 0}(-1)^{m} b_{m} \partial_{x}^{-m-1} .
$$

Since $W W^{\dagger}=1($ see $(\underline{2.5}))$, we get that $b_{m}=0$ for all $m \geq 0$. 
Theorem 2.1 There exists a function $\tau=\tau\left(x, \mathbf{t}_{\mathrm{o}}\right)$ such that

$$
\Psi\left(x, \mathbf{t}_{\mathrm{o}}, z\right)=(2 z)^{-1 / 2} \sqrt{\partial_{x} \psi^{2}\left(x, \mathbf{t}_{\mathrm{o}}, z\right)},
$$

where

$$
\psi\left(x, \mathbf{t}_{\mathrm{o}}, z\right)=e^{x z+\zeta\left(\mathbf{t}_{\mathrm{o}}, z\right)} \frac{\tau\left(x, \mathbf{t}_{\mathrm{o}}-2\left[z^{-1}\right]_{\mathrm{o}}\right)}{\tau\left(x, \mathbf{t}_{\mathrm{o}}\right)}
$$

and we use the notation

$$
\mathbf{t}_{\mathrm{o}}+j\left[z^{-1}\right]_{\mathrm{o}}:=\left\{t_{1}+\frac{j}{z}, t_{3}+\frac{j}{3 z^{3}}, t_{5}+\frac{j}{5 z^{5}}, \ldots\right\}, \quad j \in \mathbb{Z} .
$$

Definition 2.1 The function $\tau=\tau\left(x, \mathbf{t}_{\mathrm{o}}\right)$ is called the tau-function of the CKP hierarchy.

Proof of Theorem [2.1. Representing the right hand side of (2.16) in the explicit form, we see that we should prove the formula

$$
\Psi=e^{x z+\zeta\left(\mathbf{t}_{\mathrm{o}}, z\right)} G\left(x, \mathbf{t}_{\mathrm{o}}, z\right) \frac{\tau\left(x, \mathbf{t}_{\mathrm{o}}-2\left[z^{-1}\right]_{\mathrm{o}}\right)}{\tau\left(x, \mathbf{t}_{\mathrm{o}}\right)},
$$

where

$$
G(x, \mathbf{t}, z)=\left(1+z^{-1} \partial_{t_{1}} \log \frac{\tau\left(x, \mathbf{t}_{\mathrm{o}}-2\left[z^{-1}\right]_{\mathrm{o}}\right)}{\tau\left(x, \mathbf{t}_{\mathrm{o}}\right)}\right)^{1 / 2} .
$$

The proof is based on the bilinear relation (2.15). Let us represent the wave function in the form

$$
\Psi\left(x, \mathbf{t}_{\mathrm{o}}, z\right)=e^{x z+\zeta\left(\mathbf{t}_{\mathrm{o}}, z\right)} w\left(x, \mathbf{t}_{\mathrm{o}}, z\right)
$$

and set $\mathbf{t}_{\mathrm{o}}^{\prime}=\mathbf{t}_{\mathrm{o}}-2\left[a^{-1}\right]_{\mathrm{o}}$ in the bilinear relation. We have $e^{\zeta\left(2\left[a^{-1}\right]_{\mathrm{o}}, z\right)}=\frac{a+z}{a-z}$. Then the residue calculus yields

$$
w\left(\mathbf{t}_{\mathrm{o}}, a\right) w\left(\mathbf{t}_{\mathrm{o}}-2\left[a^{-1}\right]_{\mathrm{o}},-a\right)=f\left(\mathbf{t}_{\mathrm{o}}, a\right)
$$

where

$$
f\left(\mathbf{t}_{\mathrm{o}}, z\right)=1+\frac{1}{2 z}\left(\xi_{1}\left(\mathbf{t}_{\mathrm{o}}\right)-\xi_{1}\left(\mathbf{t}_{\mathrm{o}}-2\left[z^{-1}\right]_{\mathrm{o}}\right)\right)
$$

and we do not indicate the dependence on $x$ for brevity. Next, we set $\mathbf{t}_{\mathrm{o}}^{\prime}=\mathbf{t}_{\mathrm{o}}-2\left[a^{-1}\right]_{\mathrm{o}}-$ $2\left[b^{-1}\right]_{\mathrm{o}}$ in the bilinear relation and the residue calculus yields

$$
\begin{gathered}
\frac{a+b}{a-b}\left(a w\left(\mathbf{t}_{\mathrm{o}}, a\right) w\left(\mathbf{t}_{\mathrm{o}}-2\left[a^{-1}\right]_{\mathrm{o}}-2\left[b^{-1}\right]_{\mathrm{o}},-a\right)-b w\left(\mathbf{t}_{\mathrm{o}}, b\right) w\left(\mathbf{t}_{\mathrm{o}}-2\left[a^{-1}\right]_{\mathrm{o}}-2\left[b^{-1}\right]_{\mathrm{o}},-b\right)\right) \\
=a+b+\frac{1}{2}\left(\xi_{1}\left(\mathbf{t}_{\mathrm{o}}\right)-\xi_{1}\left(\mathbf{t}_{\mathrm{o}}-2\left[a^{-1}\right]_{\mathrm{o}}-2\left[b^{-1}\right]_{\mathrm{o}}\right) .\right.
\end{gathered}
$$

Using the relation (2.21), we can represent this equation in the form

$$
\begin{gathered}
\frac{1}{a-b}\left(a f\left(\mathbf{t}_{\mathrm{o}}-2\left[b^{-1}\right]_{\mathrm{o}}, a\right) \frac{w\left(\mathbf{t}_{\mathrm{o}}, a\right)}{w\left(\mathbf{t}_{\mathrm{o}}-2\left[b^{-1}\right]_{\mathrm{o}}, a\right)}-b f\left(\mathbf{t}_{\mathrm{o}}-2\left[a^{-1}\right]_{\mathrm{o}}, b\right) \frac{w\left(\mathbf{t}_{\mathrm{o}}, b\right)}{w\left(\mathbf{t}_{\mathrm{o}}-2\left[a^{-1}\right]_{\mathrm{o}}, b\right)}\right) \\
=1+\frac{\xi_{1}\left(\mathbf{t}_{\mathrm{o}}\right)-\xi_{1}\left(\mathbf{t}_{\mathrm{o}}-2\left[a^{-1}\right]_{\mathrm{o}}-2\left[b^{-1}\right]_{\mathrm{o}}\right)}{2(a+b)} .
\end{gathered}
$$


Shifting here $\mathbf{t}_{\mathrm{o}} \rightarrow \mathbf{t}_{\mathrm{o}}+2\left[b^{-1}\right]_{\mathrm{o}}$, changing the sign of $b$ (i.e, changing $b \rightarrow-b$ ) and using (2.21) in the second term in the left hand side after that, we arrive at the equation

$$
\begin{gathered}
\frac{1}{a+b}\left(a f\left(\mathbf{t}_{\mathrm{o}}, a\right) \frac{w\left(\mathbf{t}_{\mathrm{o}}-2\left[b^{-1}\right]_{\mathrm{o}}, a\right)}{w\left(\mathbf{t}_{\mathrm{o}}, a\right)}-b f\left(\mathbf{t}_{\mathrm{o}}, b\right) \frac{w\left(\mathbf{t}_{\mathrm{o}}-2\left[a^{-1}\right]_{\mathrm{o}}, b\right)}{w\left(\mathbf{t}_{\mathrm{o}}, b\right)}\right) \\
=1+\frac{\xi_{1}\left(\mathbf{t}_{\mathrm{o}}-2\left[b^{-1}\right]_{\mathrm{o}}\right)-\xi_{1}\left(\mathbf{t}_{\mathrm{o}}-2\left[a^{-1}\right]_{\mathrm{o}}\right)}{2(a-b)} .
\end{gathered}
$$

Together equations (2.24), (2.25) form the system of equations

$$
\left\{\begin{array}{l}
\frac{1}{a-b}\left(a f\left(\mathbf{t}_{\mathrm{o}}-2\left[b^{-1}\right]_{\mathrm{o}}, a\right) X_{a}^{-1}-b f\left(\mathbf{t}_{\mathrm{o}}-2\left[a^{-1}\right]_{\mathrm{o}}, b\right) X_{b}^{-1}\right)=\frac{a f\left(\mathbf{t}_{\mathrm{o}}, a\right)+b f\left(\mathbf{t}_{\mathrm{o}}-2\left[a^{-1}\right]_{\mathrm{o}}, b\right)}{a+b} \\
\frac{1}{a+b}\left(a f\left(\mathbf{t}_{\mathrm{o}}, a\right) X_{a}-b f\left(\mathbf{t}_{\mathrm{o}}, b\right) X_{b}\right)=\frac{a f\left(\mathbf{t}_{\mathrm{o}}, a\right)-b f\left(\mathbf{t}_{\mathrm{o}}, b\right)}{a-b}
\end{array}\right.
$$

for the "unknowns"

$$
X_{a}=\frac{w\left(\mathbf{t}_{\mathrm{o}}-2\left[b^{-1}\right]_{\mathrm{o}}, a\right)}{w\left(\mathbf{t}_{\mathrm{o}}, a\right)}, \quad X_{b}=\frac{w\left(\mathbf{t}_{\mathrm{o}}-2\left[a^{-1}\right]_{\mathrm{o}}, b\right)}{w\left(\mathbf{t}_{\mathrm{o}}, b\right)} .
$$

Multiplying the two equations (2.26), one obtains, using the identity

$$
a f\left(\mathbf{t}_{\mathrm{o}}, a\right)-a f\left(\mathbf{t}_{\mathrm{o}}-2\left[b^{-1}\right]_{\mathrm{o}}, a\right)-b f\left(\mathbf{t}_{\mathrm{o}}, b\right)+b f\left(\mathbf{t}_{\mathrm{o}}-2\left[a^{-1}\right]_{\mathrm{o}}, b\right)=0,
$$

the following simple relation:

$$
\frac{w\left(\mathbf{t}_{\mathrm{o}}, a\right) w\left(\mathbf{t}_{\mathrm{o}}-2\left[a^{-1}\right]_{\mathrm{o}}, b\right)}{w\left(\mathbf{t}_{\mathrm{o}}, b\right) w\left(\mathbf{t}_{\mathrm{o}}-2\left[b^{-1}\right]_{\mathrm{o}}, a\right)}=\left(\frac{f\left(\mathbf{t}_{\mathrm{o}}, a\right) f\left(\mathbf{t}_{\mathrm{o}}-2\left[a^{-1}\right]_{\mathrm{o}}, b\right)}{f\left(\mathbf{t}_{\mathrm{o}}, b\right) f\left(\mathbf{t}_{\mathrm{o}}-2\left[b^{-1}\right]_{\mathrm{o}}, a\right)}\right)^{1 / 2} .
$$

Therefore, introducing $w_{0}\left(\mathbf{t}_{\mathrm{o}}, z\right)=w\left(\mathbf{t}_{\mathrm{o}}, z\right) f^{-1 / 2}\left(\mathbf{t}_{\mathrm{o}}, z\right)$, we get

$$
\frac{w_{0}\left(\mathbf{t}_{\mathrm{o}}, a\right) w_{0}\left(\mathbf{t}_{\mathrm{o}}-2\left[a^{-1}\right]_{\mathrm{o}}, b\right)}{w_{0}\left(\mathbf{t}_{\mathrm{o}}, b\right) w_{0}\left(\mathbf{t}_{\mathrm{o}}-2\left[b^{-1}\right]_{\mathrm{o}}, a\right)}=1 \text {. }
$$

Our goal is to prove that there exists a function $\tau\left(\mathbf{t}_{\mathrm{o}}\right)$ such that

$$
w_{0}\left(\mathbf{t}_{\mathrm{o}}, z\right)=\frac{\tau\left(\mathbf{t}_{\mathrm{o}}-2\left[z^{-1}\right]_{\mathrm{o}}\right)}{\tau\left(\mathbf{t}_{\mathrm{o}}\right)} .
$$

For that it is enough to show that there is a function $\tau$ such that the equation

$$
\hat{\mathcal{D}}\left(w_{0}\left(\mathbf{t}_{\mathrm{o}}, z\right)+\tau\left(\mathbf{t}_{\mathrm{o}}\right)\right)=0
$$

with

$$
\hat{\mathcal{D}}:=\partial_{z}-2 \sum_{m \geq 1, \text { odd }} z^{-m-1} \partial_{t_{m}}
$$

holds. 
Indeed, integrating equation $\hat{\mathcal{D}} F=0$ along its characteristics we get that a function $F\left(\mathbf{t}_{\mathrm{o}}, z\right)$ is in the kernel of the differential operator $\hat{\mathcal{D}}$ if and only it is of the form

$$
F\left(\mathbf{t}_{\mathrm{o}}, z\right)=f\left(\mathbf{t}_{\mathrm{o}}-2\left[z^{-1}\right]_{\mathrm{o}}\right)
$$

for some function $f\left(\mathbf{t}_{\mathrm{o}}\right)$. For $F$ as in (2.32) the initial condition $w_{0}\left(\mathbf{t}_{\mathrm{o}}, \infty\right)=0$ allows to identify the corresponding function $f$ with $\tau$.

Equation (2.32) is equivalent to the equations

$$
Y_{n}:=\underset{z=\infty}{\operatorname{res}}\left[z^{n} \hat{\mathcal{D}} \log w_{0}\right]=2 \frac{\partial \log \tau}{\partial t_{n}}
$$

Therefore, to complete the proof of the existence of the tau-function it remains only to show that $\partial_{t_{n}} Y_{m}\left(\mathbf{t}_{\mathrm{o}}\right)=\partial_{t_{m}} Y_{n}\left(\mathbf{t}_{\mathrm{o}}\right)$.

Changing $a \rightarrow z, b \rightarrow \zeta$ in (2.30), and applying the operator $\hat{\mathcal{D}}$ to logarithm of this equality, we get

$$
\hat{\mathcal{D}}\left(\log w_{0}\left(\mathbf{t}_{\mathrm{o}}, z\right)-\log w_{0}\left(\mathbf{t}_{\mathrm{o}}-2\left[\zeta^{-1}\right]_{\mathrm{o}}, z\right)+\log w_{0}\left(\mathbf{t}_{\mathrm{o}}, \zeta\right)\right)=0,
$$

or

$$
Y_{n}\left(\mathbf{t}_{\mathrm{o}}\right)-Y_{n}\left(\mathbf{t}_{\mathrm{o}}-2\left[\zeta^{-1}\right]_{\mathrm{o}}\right)=-2 \partial_{t_{n}} \log w_{0}\left(\mathbf{t}_{\mathrm{o}}, \zeta\right)
$$

Denote $F_{m n}=\partial_{t_{m}} Y_{n}-\partial_{t_{n}} Y_{m}$. Then, from (2.34) it follows that the equation

$$
F_{m n}\left(\mathbf{t}_{\mathrm{o}}\right)=F_{m n}\left(\mathbf{t}_{\mathrm{o}}-2\left[\zeta^{-1}\right]_{\mathrm{o}}\right)
$$

holds identically in $\zeta$. Expanding the right hand side in a power series,

$$
F_{m n}\left(\mathbf{t}_{\mathrm{o}}-2\left[\zeta^{-1}\right]_{\mathrm{o}}\right)=F_{m n}\left(\mathbf{t}_{\mathrm{o}}\right)-2 \zeta^{-1} \partial_{t_{1}} F_{m n}\left(\mathbf{t}_{\mathrm{o}}\right)-\frac{2}{3} \zeta^{-3}\left(\partial_{t_{3}} F_{m n}\left(\mathbf{t}_{\mathrm{o}}\right)+2 \partial_{t_{1}}^{3} F_{m n}\left(\mathbf{t}_{\mathrm{o}}\right)\right)+\ldots,
$$

we see from the $\zeta^{-1}$-term that $F_{m n}$ does not depend on $t_{1}$. Then from the $\zeta^{-3}$-term we conclude that it does not depend on $t_{3}$ and so on, so it does not depend on $t_{k}$ for all (odd) $k: F_{m n}=2 a_{m n}$, where $a_{m n}$ are some constants such that $a_{m n}=-a_{n m}$. Therefore, we can write

$$
Y_{n}=\sum_{m} a_{m n} t_{m}+\partial_{t_{n}} h
$$

where $h=h\left(\mathbf{t}_{\mathrm{o}}\right)$ is some function. Then from (2.34) we have

$$
-2 \partial_{t_{n}} \log w_{0}\left(\mathbf{t}_{\mathrm{o}}, z\right)=\partial_{t_{n}}\left(h\left(\mathbf{t}_{\mathrm{o}}\right)-h\left(\mathbf{t}_{\mathrm{o}}-2\left[z^{-1}\right]_{\mathrm{o}}\right)\right)+2 \sum_{m \text { odd }} \frac{a_{m n}}{m} z^{-m},
$$

or, after integration,

$$
\log w_{0}\left(\mathbf{t}_{\mathrm{o}}, z\right)=\frac{1}{2} h\left(\mathbf{t}_{\mathrm{o}}-2\left[z^{-1}\right]_{\mathrm{o}}\right)-\frac{1}{2} h\left(\mathbf{t}_{\mathrm{o}}\right)-\sum_{m \text { odd }} \frac{a_{m n}}{m} z^{-m} t_{n}+\varphi(z),
$$

where $\varphi(z)$ is a function of $z$ only. Substituting this into logarithm of (2.30), we conclude that $a_{m n}=0$.

Now, writing $w\left(\mathbf{t}_{\mathrm{o}}, z\right)=f^{1 / 2}\left(\mathbf{t}_{\mathrm{o}}, z\right) w_{0}\left(\mathbf{t}_{\mathrm{o}}, z\right)$ and noting that $f\left(\mathbf{t}_{\mathrm{o}}, z\right)=1+O\left(z^{-2}\right)$, we see that

$$
\xi_{1}\left(\mathbf{t}_{\mathrm{o}}\right)=-2 \partial_{t_{1}} \log \tau\left(\mathbf{t}_{\mathrm{o}}\right)
$$


and we arrive at (2.19) with $G=f^{1 / 2}$.

Remark. The proof given above is rather involved. It is instructive to obtain (2.19) up to a common $x$-independent factor in the following easy way [8, 9]. Let us apply $\partial_{t_{1}}$ to (2.15) and set $\mathbf{t}_{\mathrm{o}}^{\prime}=\mathbf{t}_{\mathrm{o}}-2\left[a^{-1}\right]_{\mathrm{o}}$. The residue calculus yields

$$
\begin{gathered}
2 a^{2}\left(1-w\left(\mathbf{t}_{\mathrm{o}}, a\right) w\left(\mathbf{t}_{\mathrm{o}}-2\left[a^{-1}\right]_{\mathrm{o}},-a\right)\right)-2 a w^{\prime}\left(\mathbf{t}_{\mathrm{o}}, a\right) w\left(\mathbf{t}_{\mathrm{o}}-2\left[a^{-1}\right]_{\mathrm{o}},-a\right) \\
+2 a\left(\xi_{1}\left(\mathbf{t}_{\mathrm{o}}\right)-\xi_{1}\left(\mathbf{t}_{\mathrm{o}}-2\left[a^{-1}\right]_{\mathrm{o}}\right)\right)+\xi_{2}\left(\mathbf{t}_{\mathrm{o}}-2\left[a^{-1}\right]_{\mathrm{o}}\right)+\xi_{2}\left(\mathbf{t}_{\mathrm{o}}\right)+\xi_{1}^{\prime}\left(\mathbf{t}_{\mathrm{o}}\right) \\
-\xi_{1}\left(\mathbf{t}_{\mathrm{o}}\right) \xi_{1}\left(\mathbf{t}_{\mathrm{o}}-2\left[a^{-1}\right]_{\mathrm{o}}\right)=0
\end{gathered}
$$

where prime means the $x$-derivative and we again do not indicate the dependence on $x$ explicitly. Letting $a \rightarrow \infty$, we get the relation

$$
2 \xi_{2}\left(\mathbf{t}_{\mathrm{o}}\right)=\xi_{1}^{2}\left(\mathbf{t}_{\mathrm{o}}\right)-\xi_{1}^{\prime}\left(\mathbf{t}_{\mathrm{o}}\right)
$$

which also directly follows from $W W^{\dagger}=1$. Plugging it back to (2.37), we can rewrite equation (2.37) in the form

$$
w^{\prime}\left(\mathbf{t}_{\mathrm{o}}, a\right) w\left(\mathbf{t}_{\mathrm{o}}-2\left[a^{-1}\right]_{\mathrm{o}},-a\right)=a f\left(\mathbf{t}_{\mathrm{o}}, a\right)\left(f\left(\mathbf{t}_{\mathrm{o}}, a\right)-1\right)+\frac{1}{2} f^{\prime}\left(\mathbf{t}_{\mathrm{o}}, a\right) .
$$

Using (2.21), we conclude that

$$
\begin{aligned}
& \partial_{x} \log w\left(\mathbf{t}_{\mathrm{o}}, a\right)=a\left(f\left(\mathbf{t}_{\mathrm{o}}, a\right)-1\right)+\frac{1}{2} \partial_{x} \log f\left(\mathbf{t}_{\mathrm{o}}, a\right) \\
& =\frac{1}{2}\left(\xi_{1}\left(\mathbf{t}_{\mathrm{o}}\right)-\xi_{1}\left(\mathbf{t}_{\mathrm{o}}-2\left[a^{-1}\right]_{\mathrm{o}}\right)\right)+\frac{1}{2} \partial_{x} \log f\left(\mathbf{t}_{\mathrm{o}}, a\right) .
\end{aligned}
$$

Now, setting $\xi_{1}\left(x, \mathbf{t}_{\mathrm{o}}\right)=-2 \partial_{x} \log \tau\left(x, \mathbf{t}_{\mathrm{o}}\right)$ and integrating, we arrive at (2.19) with $G=$ $f^{1 / 2}$ up to a common multiplier which does not depend on $x$.

Remark. Substitution of (2.14) into (2.11) with $k=3$ gives that the function $u$ in (2.8) equals

$$
u=-\frac{1}{2} \xi_{1}^{\prime}=\partial_{x}^{2} \log \tau
$$

\subsection{CKP hierarchy versus KP hierarchy}

The goal of this section is to prove that the CKP hierarchy can be identified with the restriction of odd-times flows of the KP hierarchy onto the locus of turning points of even-times flows.

Recall that wave function $\Psi^{\mathrm{KP}}$ and the adjoint wave function $\Psi^{\dagger \mathrm{KP}}$ of the KP hierarchy are expressed through the tau-function $\tau^{\mathrm{KP}}$ as

$$
\begin{aligned}
\Psi^{\mathrm{KP}}(x, \mathbf{t} ; z) & =\exp \left(x z+\sum_{k \geq 1} t_{k} z^{k}\right) \frac{\tau^{\mathrm{KP}}\left(x, \mathbf{t}-\left[z^{-1}\right]\right)}{\tau^{\mathrm{KP}}(x, \mathbf{t})}, \\
\Psi^{\dagger \mathrm{KP}}(x, \mathbf{t} ; z) & =\exp \left(-x z-\sum_{k \geq 1} t_{k} z^{k}\right) \frac{\tau^{\mathrm{KP}}\left(x, \mathbf{t}+\left[z^{-1}\right]\right)}{\tau^{\mathrm{KP}}(x, \mathbf{t})} .
\end{aligned}
$$


where the notation (1.7) for the special shift of times is used. The origin of these expressions is the bilinear relation 5 ]

$$
\oint_{C_{\infty}} \Psi^{\mathrm{KP}}(x, \mathbf{t}, z) \Psi^{\dagger \mathrm{KP}}\left(x, \mathbf{t}^{\prime}, z\right) d z=0
$$

equivalent to (1.6) .

A direct consequence of the bilinear relation (2.44) with the wave functions given by (2.42), (2.43) is the Hirota-Miwa equation for the tau-function of the KP hierarchy

$$
\begin{aligned}
& \left(z_{1}-z_{2}\right)\left(z_{3}-z_{4}\right) \tau^{\mathrm{KP}}\left(\mathbf{t}-\left[z_{1}^{-1}\right]-\left[z_{2}^{-1}\right]\right) \tau^{\mathrm{KP}}\left(\mathbf{t}-\left[z_{3}^{-1}\right]-\left[z_{4}^{-1}\right]\right) \\
& \quad+\left(z_{2}-z_{3}\right)\left(z_{1}-z_{4}\right) \tau^{\mathrm{KP}}\left(\mathbf{t}-\left[z_{2}^{-1}\right]-\left[z_{3}^{-1}\right]\right) \tau^{\mathrm{KP}}\left(\mathbf{t}-\left[z_{1}^{-1}\right]-\left[z_{4}^{-1}\right]\right) \\
& \quad+\left(z_{3}-z_{1}\right)\left(z_{2}-z_{4}\right) \tau^{\mathrm{KP}}\left(\mathbf{t}-\left[z_{1}^{-1}\right]-\left[z_{3}^{-1}\right]\right) \tau^{\mathrm{KP}}\left(\mathbf{t}-\left[z_{2}^{-1}\right]-\left[z_{4}^{-1}\right]\right)=0 .
\end{aligned}
$$

It is a generating equation for the differential equations of the hierarchy. The differential equations are obtained by expanding it in negative powers of $z_{1}, z_{2}, z_{3}, z_{4}$. In the limit $z_{4} \rightarrow \infty, z_{3} \rightarrow \infty$ equation (2.45) becomes

$$
\begin{aligned}
& \partial_{x} \log \frac{\tau^{\mathrm{KP}}\left(x, \mathbf{t}+\left[z_{1}^{-1}\right]-\left[z_{2}^{-1}\right]\right)}{\tau^{\mathrm{KP}}(x, \mathbf{t})} \\
& =\left(z_{2}-z_{1}\right)\left(\frac{\tau^{\mathrm{KP}}\left(x, \mathbf{t}+\left[z_{1}^{-1}\right]\right) \tau^{\mathrm{KP}}\left(x, \mathbf{t}-\left[z_{2}^{-1}\right]\right)}{\tau^{\mathrm{KP}}(x, \mathbf{t}) \tau^{\mathrm{KP}}\left(x, \mathbf{t}+\left[z_{1}^{-1}\right]-\left[z_{2}^{-1}\right]\right)}-1\right) .
\end{aligned}
$$

We will need a particular case of (2.46) at $z_{2}=-z_{1}=z$ which we write in the form

$$
\frac{1}{2 z} \partial_{x}\left(e^{2 x z} \frac{\tau^{\mathrm{KP}}\left(x, \mathbf{t}+\left[-z^{-1}\right]-\left[z^{-1}\right]\right)}{\tau^{\mathrm{KP}}(x, \mathbf{t})}\right)=e^{2 x z} \frac{\tau^{\mathrm{KP}}\left(x, \mathbf{t}+\left[-z^{-1}\right]\right) \tau^{\mathrm{KP}}\left(x, \mathbf{t}-\left[z^{-1}\right]\right)}{\left(\tau^{\mathrm{KP}}(x, \mathbf{t})\right)^{2}} .
$$

The following theorem gives an expression for the CKP tau-functions in terms of the $\mathrm{KP}$ tau-functions satisfying the "turning points" constraint (1.10).

Theorem 2.2 The KP tau-function $\tau^{\mathrm{KP}}(x, \mathbf{t})$ is the KP extension of a solution of the $C K P$ hierarchy if the equation

$$
\left.\partial_{t_{2}} \log \tau^{\mathrm{KP}}\right|_{\mathbf{t}_{\mathrm{e}}=0}=0
$$

holds for all $t_{1}, t_{3}, t_{5}, \ldots$ when "even" times $\mathbf{t}_{\mathrm{e}}=\left\{t_{2}, t_{4}, t_{6}, \ldots\right\}$ are set equal to zero. Conversely, in the equivalence class of any KP tau-function corresponding to KP extension of a solution to the CKP hierarchy there is one which satisfies (2.48). Moreover, the CKP tau-function defined in Theorem 2.1 is equal to

$$
\tau\left(x, \mathbf{t}_{\mathrm{o}}\right)=\sqrt{\tau^{\mathrm{KP}}\left(x, t_{1}, 0, t_{3}, 0, \ldots\right)} .
$$


Proof. Comparing (2.44) and (2.15), we see that the wave functions of the CKP and KP hierarchies are related as

$$
\begin{aligned}
& \Psi\left(x, \mathbf{t}_{\mathrm{o}}, z\right)=e^{\chi(z)} \Psi^{\mathrm{KP}}\left(x, t_{1}, 0, t_{3}, 0, \ldots, z\right), \\
& \Psi\left(x, \mathbf{t}_{\mathrm{o}},-z\right)=e^{-\chi(z)} \Psi^{\dagger \mathrm{KP}}\left(x, t_{1}, 0, t_{3}, 0, \ldots, z\right)
\end{aligned}
$$

with some function $\chi(z)$ such that $\chi(\infty)=0$, i.e.

$$
\Psi^{\dagger \mathrm{KP}}\left(x, t_{1}, 0, t_{3}, 0, \ldots, z\right)=e^{2 \chi_{\mathrm{e}}(z)} \Psi^{\mathrm{KP}}\left(x, t_{1}, 0, t_{3}, 0, \ldots,-z\right),
$$

where $\chi_{\mathrm{e}}(z)=\frac{1}{2}(\chi(z)+\chi(-z))$ is the even part of the function $\chi(z)$. From (2.42), (2.43) and (2.50) it follows that the KP tau-function is the extension of a solution of the CKP hierarchy if and only if the equation

$$
\begin{aligned}
\tau^{\mathrm{KP}}\left(x, t_{1}+z^{-1}\right. & \left., \frac{1}{2} z^{-2}, t_{3}+\frac{1}{3} z^{-3}, \frac{1}{4} z^{-4}, \ldots\right) \\
& =e^{2 \chi_{\mathrm{e}}(z)} \tau^{\mathrm{KP}}\left(x, t_{1}+z^{-1},-\frac{1}{2} z^{-2}, t_{3}+\frac{1}{3} z^{-3},-\frac{1}{4} z^{-4}, \ldots\right)
\end{aligned}
$$

holds identically for all $z, x, t_{1}, t_{3}, t_{5}, \ldots$. Shifting the odd times, we can rewrite this condition as

$$
\log \tau^{\mathrm{KP}}\left(x, t_{1}, \frac{1}{2} z^{-2}, t_{3}, \frac{1}{4} z^{-4}, \ldots\right)-\log \tau^{\mathrm{KP}}\left(x, t_{1},-\frac{1}{2} z^{-2}, t_{3},-\frac{1}{4} z^{-4}, \ldots\right)=2 \chi_{\mathrm{e}}(z) .
$$

Comparing the coefficients at $z^{-2}$ of the expansions of the left and right hand sides of (2.52) and passing to an equivalent tau-function if necessary, we get (2.48), i.e. the "only if" part of the theorem statement is proven.

We begin the proof of the "if part" by the following lemma.

Lemma 2.1 On solutions of the KP hierarchy equation (2.48) implies that all derivatives of odd degree higher then 1 with respect to various even times are equal to zero for all $x, \mathbf{t}_{\mathrm{o}}$, i.e.

$$
\left.\partial_{t_{2 k_{1}}} \partial_{t_{2 k_{2}}} \ldots \partial_{t_{2 k_{2 m+1}}} \log \tau^{\mathrm{KP}}\right|_{\mathbf{t}_{\mathrm{e}}=0}=0
$$

for all $k_{1}, k_{2}, \ldots, k_{2 m+1} \geq 1, m \geq 1$. Besides, first order derivatives with respect to even times satisfy

$$
\left.\partial_{x} \partial_{t_{4}} \log \tau^{\mathrm{KP}}\right|_{\mathbf{t}_{\mathrm{e}}=0}=\left.\partial_{x} \partial_{t_{6}} \log \tau^{\mathrm{KP}}\right|_{\mathbf{t}_{\mathrm{e}}=0}=\ldots=0 .
$$

The proof is given in Appendix A. From equations (2.54) we see that

$$
\left.\partial_{t_{2 k}} \log \tau^{\mathrm{KP}}\right|_{\mathbf{t}_{\mathrm{e}}=0}=\chi_{2 k}\left(t_{3}, t_{5}, \ldots\right)
$$

does not depend on $x$. Equation (2.48) means that $\chi_{2}=0$. Next, from (2.53) we conclude that

$$
\log \tau^{\mathrm{KP}}\left(x, t_{1}, t_{2}, t_{3}, t_{4}, \ldots\right)-\log \tau^{\mathrm{KP}}\left(x, t_{1},-t_{2}, t_{3},-t_{4}, \ldots\right)=2 \sum_{k \geq 2} \chi_{2 k}\left(t_{3}, t_{5}, \ldots\right) t_{2 k}
$$

is a linear function of $\mathbf{t}_{\mathbf{e}}$. Therefore, we can write

$$
\tau^{\mathrm{KP}}\left(x, t_{1}, \frac{1}{2} z^{-2}, t_{3}, \frac{1}{4} z^{-4}, \ldots\right)=e^{2 \chi_{\mathrm{e}}\left(t_{3}, t_{5}, \ldots ; z\right)} \tau^{\mathrm{KP}}\left(x, t_{1},-\frac{1}{2} z^{-2}, t_{3},-\frac{1}{4} z^{-4}, \ldots\right),
$$


where $\chi_{\mathrm{e}}\left(t_{3}, t_{5}, \ldots ; z\right)$ is a function of the times $t_{3}, t_{5}, \ldots$ and an even function of $z$. In its turn, (2.55) implies

$$
\Psi^{\dagger \mathrm{KP}}(x, \dot{\mathbf{t}}, z)=C\left(t_{3}+\frac{1}{3} z^{-3}, t_{5}+\frac{1}{5} z^{-5}, \ldots ; z\right) \Psi^{\mathrm{KP}}(x, \dot{\mathbf{t}},-z),
$$

where $C\left(t_{3}, t_{5}, \ldots ; z\right)=e^{\chi_{\mathrm{e}}\left(t_{3}, t_{5}, \ldots ; z\right)}$ and we use the short-hand notation

$$
\dot{\mathbf{t}}=\left\{t_{1}, 0, t_{3}, 0, \ldots\right\}
$$

(In this notation equation (2.51) takes the form $\tau^{\mathrm{KP}}\left(x, \dot{\mathbf{t}}+\left[z^{-1}\right]\right)=\tau^{\mathrm{KP}}\left(x, \dot{\mathbf{t}}-\left[-z^{-1}\right]\right)$.)

The adjoint wave function $\Psi^{\dagger \mathrm{KP}}$ satisfies the adjoint linear equation (see the independent proof in the next section), which restricted to the locus $\dot{\mathbf{t}}$ where $B_{k}^{\dagger}=-B_{k}$ for odd $k$ coincides with the linear equation for $\Psi^{\mathrm{KP}}$, so we simultaneously have

$$
\begin{aligned}
& \partial_{t_{k}} \Psi^{\dagger \mathrm{KP}}(x, \dot{\mathbf{t}}, z)=B_{k} \Psi^{\dagger \mathrm{KP}}(x, \dot{\mathbf{t}}, z), \\
& \partial_{t_{k}} \Psi^{\mathrm{KP}}(x, \dot{\mathbf{t}}, z)=B_{k} \Psi^{\mathrm{KP}}(x, \dot{\mathbf{t}}, z) .
\end{aligned}
$$

for odd $k$. Substituting (2.56) into the first of these equations, we get, after the change $z \rightarrow-z$

$$
\partial_{t_{k}} \Psi^{\mathrm{KP}}(x, \dot{\mathbf{t}}, z)+\partial_{t_{k}} \log C\left(t_{3}-\frac{1}{3} z^{-3}, t_{5}-\frac{1}{5} z^{-5}, \ldots ; z\right)=B_{k} \Psi^{\mathrm{KP}}(x, \dot{\mathbf{t}}, z),
$$

and from the second equation in (2.57) we conclude that

$$
\partial_{t_{k}} \log C\left(t_{3}-\frac{1}{3} z^{-3}, t_{5}-\frac{1}{5} z^{-5}, \ldots ; z\right)=0,
$$

i.e. $\chi_{\mathrm{e}}\left(t_{3}, t_{5}, \ldots ; z\right)=\chi_{\mathrm{e}}(z)$ is an even function of $z$ which does not depend on the times. (This function can be eliminated in (2.55) by passing to an equivalent tau-function.) Therefore, the equation (2.51) which guarantees that $\tau^{\mathrm{KP}}$ is the KP extension of a solution to the CKP hierarchy is proved.

Remark. Passing to an equivalent tau-function using the transformation (1.8), one obtains the condition $\left.\partial_{t_{2}} \log \tau^{\mathrm{KP}}\right|_{\mathbf{t}_{\mathrm{e}}}=\gamma_{2}$ instead of (2.48) . Conversely, if $\left.\partial_{t_{2}} \log \tau^{\mathrm{KP}}\right|_{\mathbf{t}_{\mathrm{e}}}=$ $\gamma_{2}$ with some nonzero $\gamma_{2}$, it is possible to pass to an equivalent tau-function satisfying (2.48) by a transformation of the form (1.8).

In order to prove that $\tau=\sqrt{\tau^{\mathrm{KP}}}([10])$ we compare two expressions for the wave function $\Psi$ of the CKP hierarchy. The first one is in terms of the KP tau-function (satisfying (2.48) ),

$$
\Psi^{\mathrm{KP}}=e^{x z+\zeta\left(\mathbf{t}_{\mathrm{o}}, z\right)} \frac{\tau^{\mathrm{KP}}\left(x, t_{1}-z^{-1},-\frac{1}{2} z^{-2}, t_{3}-\frac{1}{3} z^{-3},-\frac{1}{4} z^{-4}, \ldots\right)}{\tau^{\mathrm{KP}}\left(t_{1}, 0, t_{3}, 0, \ldots\right)},
$$

and the second one (2.19) is in terms of the CKP tau-function $\tau$. Recall that

$$
\Psi=z^{-1 / 2} \sqrt{\partial_{x} \log \psi} \cdot \psi=(2 z)^{-1 / 2} \sqrt{\partial_{x} \psi^{2}}
$$

$($ see $(2.16))$, where

$$
\psi=e^{x z+\zeta\left(\mathbf{t}_{\mathrm{o}}, z\right)} \frac{\tau\left(x, \mathbf{t}_{\mathrm{o}}-2\left[z^{-1}\right]_{\mathrm{o}}\right)}{\tau\left(x, \mathbf{t}_{\mathrm{o}}\right)} .
$$


Comparing (2.59) and (2.58), we get the equation

$$
\frac{1}{2 z} \partial_{x}\left(e^{2 x z} \frac{\tau^{2}\left(x, \mathbf{t}_{\mathrm{o}}-2\left[z^{-1}\right]_{\mathrm{o}}\right)}{\tau^{2}\left(x, \mathbf{t}_{\mathrm{o}}\right)}\right)=e^{2 x z}\left(\frac{\tau^{\mathrm{KP}}\left(x, \dot{\mathbf{t}}-\left[z^{-1}\right]\right)}{\tau^{\mathrm{KP}}(x, \dot{\mathbf{t}})}\right)^{2},
$$

where we again use the short-hand notation $\dot{\mathbf{t}}=\left\{t_{1}, 0, t_{3}, 0, \ldots\right\}$. Then using equation (2.47) we get that (2.61) is equivalent to the differential equation

$$
\partial_{x} \varphi=-2 z \varphi
$$

where

$$
\varphi=\frac{\tau^{2}\left(x, \mathbf{t}_{\mathrm{o}}-2\left[z^{-1}\right]_{\mathrm{o}}\right)}{\tau^{2}\left(x, \mathbf{t}_{\mathrm{o}}\right)}-\frac{\tau^{\mathrm{KP}}\left(x, \dot{\mathbf{t}}-2\left[z^{-1}\right]_{\mathrm{o}}\right)}{\tau^{\mathrm{KP}}(x, \dot{\mathbf{t}})} .
$$

In (2.63),$\dot{\mathbf{t}}-2\left[z^{-1}\right]_{\mathrm{o}}=\left\{t_{1}-2 z^{-1}, 0, t_{3}-\frac{2}{3} z^{-3}, 0, \ldots\right\}$. The general solution of the differential equation (2.62) is

$$
\varphi=c\left(z, t_{3}, t_{5}, \ldots\right) e^{-2\left(x+t_{1}\right) z}
$$

but from (2.63) it follows that $\varphi$ is expanded in a power series as $\varphi=\varphi_{1} z^{-1}+\varphi_{2} z^{-2}+\ldots$ as $z \rightarrow \infty$, and this means that $c$ must be equal to 0 . Therefore, $\varphi=0$, i.e.

$$
\frac{\tau^{2}\left(x, \mathbf{t}_{\mathrm{o}}-2\left[z^{-1}\right]_{\mathrm{o}}\right)}{\tau^{2}\left(x, \mathbf{t}_{\mathrm{o}}\right)}=\frac{\tau^{\mathrm{KP}}\left(x, \dot{\mathbf{t}}-2\left[z^{-1}\right]_{\mathrm{o}}\right)}{\tau^{\mathrm{KP}}(x, \dot{\mathbf{t}})}
$$

for all $z$. This is an identity on solutions to the KP/CKP hierarchies. It follows from (2.64) that $\tau^{\mathrm{KP}}=$ const $\cdot \tau^{2}$, i.e. $\tau\left(x, \mathbf{t}_{\mathrm{o}}\right)=\sqrt{\tau^{\mathrm{KP}}(x, \dot{\mathbf{t}})}$ is a tau-function of the CKP hierarchy.

Remark. Equation (2.61) is the CKP analog of the BKP statement that the correspond$\operatorname{ing} \tau^{\mathrm{KP}}$ is a full square, i.e. $\tau=\sqrt{\tau^{\mathrm{KP}}}$ is an entire function of its variables. In the CKP case $\partial_{x} \psi^{2}$ is a full square.

\section{Algebraic-geometrical solutions to the KP and CKP hierarchies}

The algebraic-geometrical construction of quasi-periodic solutions to the CKP hierarchy briefly outlined in [7] is a reduction of the algebraic-geometrical construction of solutions to the KP hierarchy proposed in [12, 13]. The main goal of this section is to give a pure algebraic-geometrical proof of an identity for the Riemann theta-function of a curve with involution having at least one fixed point. This identity is an algebraic-geometrical incarnation of the relations between KP and CKP tau-functions discussed in Section 2. 


\subsection{Prelimineries}

Let $\Gamma$ be a smooth compact algebraic curve of genus $g$. We fix a canonical basis of cycles $a_{\alpha}, b_{\alpha}(\alpha=1, \ldots, g)$ with the intersections $a_{\alpha} \circ a_{\beta}=b_{\alpha} \circ b_{\beta}=0, a_{\alpha} \circ b_{\beta}=\delta_{\alpha \beta}$ and a basis of holomorphic differentials $d \omega_{\alpha}$ normalized by the condition $\oint_{a_{\alpha}} d \omega_{\beta}=\delta_{\alpha \beta}$. The period matrix is defined as

$$
T_{\alpha \beta}=\oint_{b_{\alpha}} d \omega_{\beta}, \quad \alpha, \beta=1, \ldots, g .
$$

It is a symmetric matrix with positively defined imaginary part. The Riemann thetafunction is defined by the series

$$
\theta(\vec{z})=\theta(\vec{z} \mid T)=\sum_{\vec{n} \in \mathbb{Z}^{g}} e^{\pi i(\vec{n}, T \vec{n})+2 \pi i(\vec{n}, \vec{z})}
$$

where $\vec{z}=\left(z_{1}, \ldots, z_{g}\right)$ and $(\vec{n}, \vec{z})=\sum_{\alpha=1}^{g} n_{\alpha} z_{\alpha}$.

The Jacobian of the curve $\Gamma$ is the $g$-dimensional complex torus

$$
J(\Gamma)=\mathbb{C}^{g} /\{2 \pi i \vec{N}+2 \pi i T \vec{M}\}
$$

where $\vec{N}, \vec{M}$ are $g$-dimensional vectors with integer components. Fix a point $Q_{0} \in \Gamma$ and define the Abel map $\vec{A}(P), P \in \Gamma$ from $\Gamma$ to $J(\Gamma)$, as

$$
\vec{A}(P)=\vec{\omega}(P)=\int_{Q_{0}}^{P} d \vec{\omega}, \quad d \vec{\omega}=\left(d \omega_{1}, \ldots, d \omega_{g}\right)
$$

The Abel map can be extended to the group of divisors $\mathcal{D}=n_{1} Q_{1}+\ldots+n_{K} Q_{K}$ as

$$
\vec{A}(\mathcal{D})=\sum_{i=1}^{K} n_{i} \int_{Q_{0}}^{Q_{i}} d \vec{\omega}=\sum_{i=1}^{K} n_{i} \vec{A}\left(Q_{i}\right)
$$

Let $P_{\infty} \in \Gamma$ be a marked point and $k^{-1}$ a local parameter in a neighborhood of the marked point $\left(k=\infty\right.$ at $\left.P_{\infty}\right)$. Let $d \Omega_{j}$ be abelian differentials of the second kind with the only pole at $P_{\infty}$ of the form

$$
d \Omega_{j}=d k^{j}+O\left(k^{-2}\right) d k, \quad k \rightarrow \infty
$$

normalized by the condition $\oint_{a_{\alpha}} d \Omega_{j}=0$, and $\Omega_{j}$ be the (multi-valued) functions

$$
\Omega_{j}(P)=\int_{Q_{0}}^{P} d \Omega_{j}+q_{j}
$$

where the constants $q_{j}$ are chosen in such a way that $\Omega_{i}(P)=k^{i}+O\left(k^{-1}\right)$, namely,

$$
\Omega_{i}(P)=k^{i}+\sum_{j \geq 1} \frac{1}{j} \Omega_{i j} k^{-j}
$$

The Riemann identity implies that the matrix $\Omega_{i j}$ is symmetric: $\Omega_{i j}=\Omega_{j i}$. 
Set

$$
U_{j}^{\alpha}=\frac{1}{2 \pi i} \oint_{b_{\alpha}} d \Omega_{j}, \quad \vec{U}_{j}=\left(U_{j}^{1}, \ldots, U_{j}^{g}\right) .
$$

One can prove the following relation:

$$
\overrightarrow{d \omega}=\sum_{j \geq 1} \vec{U}_{j} k^{-j-1} d k
$$

or

$$
\vec{A}(P)-\vec{A}\left(P_{\infty}\right)=\int_{P_{\infty}}^{P} d \vec{\omega}=-\sum_{j \geq 1} \frac{1}{j} \vec{U}_{j} k^{-j}
$$

We will also use the following fact [25, 26]: for any non-special effective divisor $\mathcal{D}=$ $Q_{1}+\ldots+Q_{g}$ of degree $g$ the function

$$
f(P)=\theta(\vec{A}(P)-\vec{A}(\mathcal{D})-\vec{K})
$$

has exactly $g$ zeros at the points $Q_{1}, \ldots, Q_{g}$. Here $\vec{K}=\left(K_{1}, \ldots, K_{g}\right)$ is the vector of Riemann's constants

$$
K_{\alpha}=\pi i+\pi i T_{\alpha \alpha}-2 \pi i \sum_{\beta \neq \alpha} \oint_{a_{\beta}} \omega_{\alpha}(P) d \omega_{\beta}(P) .
$$

Let $\mathcal{K}$ be the canonical class of divisors (the equivalence class of divisors of poles and zeros of abelian differentials on $\Gamma$ ), then one can show that

$$
2 \vec{K}=-\vec{A}(\mathcal{K})
$$

It is known that $\operatorname{deg} \mathcal{K}=2 g-2$. In particular, this means that holomorphic differentials have $2 g-2$ zeros on $\Gamma$.

We also need the bi-differential $d_{P} d_{Q} \Omega(P, Q)$ such that it is symmetric in $P, Q$, its only singularity is a second order pole at $P=Q$ and the integrals over $a$-cycles vanish. It is related to the differentials $d \Omega_{j}$ as follows:

$$
\underset{Q=P_{\infty}}{\operatorname{res}}\left(k^{i}(Q) d_{P} d_{Q} \Omega(P, Q)\right)=-d \Omega_{i}(P)
$$

The expansion in the local parameters is

$$
d_{P} d_{Q} \Omega(P, Q)=\left(\frac{1}{\left(k^{-1}(P)-k^{-1}(Q)\right)^{2}}-\sum_{i, j \geq 1} \Omega_{i j} k^{1-i}(P) k^{1-j}(Q)\right) d k^{-1}(P) d k^{-1}(Q) .
$$

In fact this bi-differential can be expressed in terms of the odd theta-function

$$
\theta_{*}(\vec{z})=\theta\left[\begin{array}{l}
\vec{\delta}^{\prime} \\
\vec{\delta}^{\prime \prime}
\end{array}\right](\vec{z})=\sum_{\vec{n} \in \mathbb{Z}^{g}} e^{\pi i\left(\vec{n}+\vec{\delta}^{\prime}, T\left(\vec{n}+\vec{\delta}^{\prime}\right)\right)+2 \pi i\left(\vec{n}+\vec{\delta}^{\prime}, \vec{z}+\vec{\delta}^{\prime \prime}\right)}
$$

where $\left(\overrightarrow{\delta^{\prime}}, \vec{\delta}^{\prime \prime}\right)$ is a non-singular odd theta-characteristics. One has:

$$
d_{P} d_{Q} \Omega(P, Q)=d_{P} d_{Q} \log \theta_{*}(\vec{A}(P)-\vec{A}(Q))
$$


Calculating the double integral

$$
\int_{P_{\infty}}^{P_{1}} \int_{Q_{0}}^{P_{2}} d_{P} d_{Q} \Omega(P, Q)
$$

in two ways (using first (3.14) and then (3.15)), we obtain the equality

$$
\begin{gathered}
\log \frac{\left(k^{-1}\left(P_{1}\right)-k^{-1}\left(P_{2}\right)\right) k^{-1}\left(Q_{0}\right)}{\left(k^{-1}\left(P_{1}\right)-k^{-1}\left(Q_{0}\right)\right) k^{-1}\left(P_{2}\right)}-\sum_{i, j \geq 1} \Omega_{i j} \frac{k^{-i}\left(P_{1}\right) k^{-j}\left(P_{2}\right)}{i j}+\sum_{i, j \geq 1} \Omega_{i j} \frac{k^{-i}\left(P_{1}\right) k^{-j}\left(Q_{0}\right)}{i j} \\
=\log \frac{\theta_{*}\left(\vec{A}\left(P_{2}\right)-\vec{A}\left(P_{1}\right)\right) \theta_{*}\left(\vec{A}\left(P_{\infty}\right)\right)}{\theta_{*}\left(\vec{A}\left(P_{2}\right)-\vec{A}\left(P_{\infty}\right)\right) \theta_{*}\left(\vec{A}\left(P_{1}\right)\right)}
\end{gathered}
$$

Tending here $Q_{0} \rightarrow P_{\infty}$, we arrive at the important relation

$$
\exp \left(-\sum_{i, j \geq 1} \Omega_{i j} \frac{k_{1}^{-i} k_{2}^{-j}}{i j}\right)=\frac{C \theta_{*}\left(\vec{A}\left(P_{1}\right)-\vec{A}\left(P_{2}\right)\right)}{\left(k_{1}-k_{2}\right) \theta_{*}\left(\vec{A}\left(P_{1}\right)-\vec{A}\left(P_{\infty}\right)\right) \theta_{*}\left(\vec{A}\left(P_{2}\right)-\vec{A}\left(P_{\infty}\right)\right)}
$$

where

$$
C=\sum_{\alpha=1}^{g} U_{1}^{\alpha} \theta_{*, \alpha}(\overrightarrow{0}), \quad \theta_{*, \alpha}(\overrightarrow{0})=\left.\frac{\partial \theta_{*}(\vec{z})}{\partial z_{\alpha}}\right|_{\vec{z}=0}
$$

is a constant and $k_{1}=k\left(P_{1}\right), k_{2}=k\left(P_{2}\right)$. In particular, tending $k_{1} \rightarrow k_{2}$, we get

$$
\exp \left(-\sum_{i, j \geq 1} \Omega_{i j} \frac{k^{-i-j}}{i j}\right) d k=\frac{C d \zeta}{\theta_{*}^{2}\left(\vec{A}(P)-\vec{A}\left(P_{\infty}\right)\right)}
$$

where $d \zeta$ is the holomorphic differential

$$
d \zeta=\sum_{\alpha=1}^{g} \theta_{*, \alpha}(\overrightarrow{0}) d \omega_{\alpha}
$$

As is explained in [26], the differential $d \zeta$ has double zeros at $g-1$ points $R_{1}, \ldots, R_{g-1}$ while the function

$$
f_{*}(P)=\theta_{*}\left(\vec{A}(P)-\vec{A}\left(P_{\infty}\right)\right)
$$

has simple zeros at the same points $R_{i}$ and $P_{\infty}$. Therefore, the differential in the right hand side of (3.17) has the only (second order) pole at $P_{\infty}$ and no zeros. However, this differential is well-defined only on a covering of the curve $\Gamma$ because it is not single-valued.

Finally, we mention the trisecant Fay identity [25]:

$$
\begin{aligned}
& \theta_{*}\left(\vec{A}\left(P_{1}\right)-\vec{A}\left(P_{2}\right)\right) \theta_{*}\left(\vec{A}\left(P_{3}\right)-\vec{A}\left(P_{4}\right)\right) \theta\left(\vec{z}+\vec{A}\left(P_{1}\right)+\vec{A}\left(P_{2}\right)\right) \theta\left(\vec{z}+\vec{A}\left(P_{3}\right)+\vec{A}\left(P_{4}\right)\right) \\
& +\theta_{*}\left(\vec{A}\left(P_{2}\right)-\vec{A}\left(P_{3}\right)\right) \theta_{*}\left(\vec{A}\left(P_{1}\right)-\vec{A}\left(P_{4}\right)\right) \theta\left(\vec{z}+\vec{A}\left(P_{2}\right)+\vec{A}\left(P_{3}\right)\right) \theta\left(\vec{z}+\vec{A}\left(P_{1}\right)+\vec{A}\left(P_{4}\right)\right) \\
& +\theta_{*}\left(\vec{A}\left(P_{3}\right)-\vec{A}\left(P_{1}\right)\right) \theta_{*}\left(\vec{A}\left(P_{2}\right)-\vec{A}\left(P_{4}\right)\right) \theta\left(\vec{z}+\vec{A}\left(P_{1}\right)+\vec{A}\left(P_{3}\right)\right) \theta\left(\vec{z}+\vec{A}\left(P_{2}\right)+\vec{A}\left(P_{4}\right)\right)=0 .
\end{aligned}
$$




\subsection{The Baker-Akhiezer function}

Let $x, t_{1}, t_{2}, t_{3}, \ldots$ be a set of complex parameters (here we assume that only a finite number of them are different from zero) and let $\Gamma$ be a smooth genus $g$ algebraic curve with fixed local coordinate $k^{-1}(P)$ in the neighborhood of a fixed point $P_{\infty}$.

Lemma 3.1 ([12, 13]) Let $\mathcal{D}=Q_{1}+\ldots+Q_{g}$ be an effective non-special divisor of degree $g$. Then there is the unique function $\Psi_{B A}(x, \mathbf{t}, P)$ such that:

$1^{0}$. As a function of $P \in \Gamma$ it is meromorphic away from the marked point $P_{\infty}$ with poles at the points $Q_{s}$ of multiplicity not greater then the multiplicity of $Q_{s}$ in $\mathcal{D}$.

$2^{0}$. In the neighborhood of $P_{\infty}$ it has the form

$$
\Psi_{B A}=\exp \left(x k+\sum_{j \geq 1} t_{j} k^{j}\right)\left(1+\xi_{1} k^{-1}+\xi_{2} k^{-2}+\ldots\right), \quad k=k(P) .
$$

The function $\Psi_{B A}$ is called (one-point) Baker-Akhiezer function.

An easy corollary of the uniqueness of the Baker-Akhiezer function is

Theorem 3.1 ([12, 13]]) Let $\Psi_{B A}$ be the Baker-Akhiezer function defined by Lemma 3.1. Then for each $j=1,2,3, \ldots$ there is a unique differential operator $B_{j}$ such that the equation

$$
\left(\partial_{t_{j}}-B_{j}\right) \Psi_{B A}=0
$$

holds.

The operators $B_{j}$ above can be easy expressed in terms of the dressing operator $W$ for the Baker-Akhiezer function. Namely, the infinite series (4.9) can be represented as

$$
\Psi_{B A}=W \exp \left(x k+\sum_{j \geq 1} t_{j} k^{j}\right)
$$

where $W$ is of the form (2.3). The corresponding Lax operator of the KP hierarchy is $\mathcal{L}=W \partial_{x} W^{-1}$. By the definition we have

$$
\mathcal{L} \Psi_{B A}=k \Psi_{B A}
$$

The operator $B_{j}$ in Theorem 3.1 was defined as the unique monic order $j$ operator such that the congruence

$$
\left(k^{j}-B_{j}\right) \Psi_{B A}=O(1 / k) \exp \left(x k+\sum_{j \geq 1} t_{j} k^{j}\right)
$$

holds. Using (3.23) , it is easy to identify $B_{j}=\mathcal{L}_{+}^{j}$. Indeed

$$
\left(k^{j}-\mathcal{L}_{+}^{j}\right) \Psi_{B A}=\left(\mathcal{L}^{j}-\mathcal{L}_{+}^{j}\right) \Psi_{B A}=\mathcal{L}_{-}^{j} \Psi_{B A}=O(1 / k) \exp \left(x k+\sum_{j \geq 1} t_{j} k^{j}\right)
$$

The compatibility conditions of equations (3.21) imply 
Corollary 3.1 The operators $B_{j}$ defined by the $B A$ function satisfies the equations

$$
\left[\partial_{t_{j}}-B_{j}, \partial_{t_{l}}-B_{l}\right]=0 .
$$

It is the Zakharov-Shabat form (1.2) of the KP hierarchy. Note that equation (3.21) implies the evolution equation for the dressing operator:

$$
\partial_{t_{j}} W=-\left(W \partial_{x}^{j} W^{-1}\right)_{-} W
$$

where $(\ldots)_{-}$is the projection to negative powers of the operator $\partial_{x}$.

\subsection{The dual Baker-Akhiezer function}

For further comparison with the tau-functional formulation of the KP hierarchy let us present the notion of the dual (adjoint) Baker-Akhiezer function introduced in [27] (see the details in [2, 28]).

First we define duality for divisors of degree $g$. For a generic effective degree $g$ divisor $\mathcal{D}=Q_{1}+\ldots+Q_{g}$ there is a unique up to a constant factor the abelian differential $d \Omega$ with the only (second order) pole at $P_{\infty}$ vanishing (with the corresponding multiplicity) at the points $Q_{s}$. The zero divisor of $d \Omega$ is of degree $2 g$. Hence, it has other $g$ zeros at some points $Q_{1}^{\dagger}, \ldots, Q_{g}^{\dagger}$. The divisor

$$
\mathcal{D}^{\dagger}=Q_{1}^{\dagger}+\ldots+Q_{g}^{\dagger}
$$

is called dual to $\mathcal{D}$. By the definition we have the equality

$$
\mathcal{D}+\mathcal{D}^{\dagger}=\mathcal{K}+2 P_{\infty}
$$

(where $\mathcal{K}$ is the canonical class), which under the Abel transform takes the form

$$
\vec{A}(\mathcal{D})+\vec{A}\left(\mathcal{D}^{\dagger}\right)+2 \vec{K}-2 \vec{A}\left(P_{\infty}\right)=0
$$

The dual (adjoint) Baker-Akhiezer function $\Psi_{B A}^{\dagger}$ has the divisor of poles $\mathcal{D}^{\dagger}$ and in the vicinity of $P_{\infty}$ it has the form

$$
\Psi_{B A}^{\dagger}=\exp \left(-x k-\sum_{j \geq 1} t_{j} k^{j}\right)\left(1+\xi_{1}^{\dagger} k^{-1}+\xi_{2}^{\dagger} k^{-2}+\ldots\right) .
$$

The differential $\Psi_{B A}(x, \mathbf{t}, P) \Psi_{B A}^{\dagger}\left(x, \mathbf{t}^{\prime}, P\right) d \Omega(P)$, where we have denoted the set of times as $\mathbf{t}=\left\{t_{1}, t_{2}, t_{3}, \ldots\right\}$ for brevity, is holomorphic everywhere on $\Gamma$ except the point $P_{\infty}$ (because poles of the Baker-Akhiezer functions are canceled by zeros of $d \Omega$ ). Therefore, its "residue" at this point is equal to zero, i.e.,

$$
\oint_{C_{\infty}} \Psi_{B A}(x, \mathbf{t}, P) \Psi_{B A}^{\dagger}\left(x, \mathbf{t}^{\prime}, P\right) d \Omega(P)=0
$$

for all $\mathbf{t}, \mathbf{t}^{\prime}$, where $C_{\infty}$ is a small contour around the point $P_{\infty}$. Equation (3.29) is equivalent to the equation

$$
\operatorname{res}_{P_{\infty}}\left(\partial_{x}^{i} \Psi_{B A}(x, \mathbf{t}, P) \Psi_{B A}^{\dagger}(x, \mathbf{t}, P)\right) d \Omega(P)=0, \quad i=1,2,3, \ldots
$$

from which one can derive the following theorem (see [2] and [28] for more details). 
Theorem 3.2 The dual Baker-Akhiezer function is equal to

$$
\Psi_{B A}^{\dagger}=\left(W^{\dagger}\right)^{-1} \exp \left(-x k-\sum_{j \geq 1} t_{j} k^{j}\right)
$$

and satisfies the adjoint equations

$$
\mathcal{L}^{\dagger} \Psi_{B A}^{\dagger}=k \Psi_{B A}^{\dagger}, \quad-\partial_{t_{j}} \Psi_{B A}^{\dagger}=B_{j}^{\dagger} \Psi_{B A}^{\dagger} .
$$

For completeness we outline here a direct proof of the theorem. Equations (3.32) immediately follow from (3.25) and (3.31):

$$
\begin{gathered}
-\partial_{t_{j}} \Psi_{B A}^{\dagger}=\left(k^{j}\left(W^{\dagger}\right)^{-1}-\left(W^{\dagger}\right)^{-1} \partial_{t_{j}} W^{\dagger}\left(W^{\dagger}\right)^{-1}\right) \exp \left(-x k-\sum_{j \geq 1} t_{j} k^{j}\right) \\
=\left(k^{j}\left(W^{\dagger}\right)^{-1}-\left(\left(W^{\dagger}\right)^{-1}\left(-\partial_{x}\right)^{j} W^{\dagger}\right)_{-}\left(W^{\dagger}\right)^{-1}\right) \exp \left(-x k-\sum_{j \geq 1} t_{j} k^{j}\right) \\
=\left(\left(\mathcal{L}^{\dagger}\right)^{j}-\left(\mathcal{L}^{\dagger}\right)_{-}^{j}\right) \Psi_{B A}^{\dagger}=B_{j}^{\dagger} \Psi_{B A}^{\dagger} .
\end{gathered}
$$

In order to prove (3.31) we note that equation (3.29) written in the local parameter $k$ implies

$$
b_{m}=\left.\frac{1}{2 \pi i} \partial_{x^{\prime}}^{m} \oint_{C_{\infty}} \Psi_{B A}(x, \mathbf{t}, k) \Psi_{B A}^{\dagger}\left(x^{\prime}, \mathbf{t}, k\right) \varphi(k) \frac{d k}{2 \pi i}\right|_{x^{\prime}=x}=0 \quad \text { for all } m \geq 0 .
$$

Here

$$
\varphi(k)=\frac{d \Omega}{d k}=\sum_{j \geq 0} \varphi_{j} k^{-j}
$$

We set

$$
\Psi_{B A}^{\dagger}=V \exp \left(-x k-\sum_{j \geq 1} t_{j} k^{j}\right), \quad V=1+\xi_{1}^{\dagger} \partial_{x}^{-1}+\xi_{2}^{\dagger} \partial_{x}^{-2}+\ldots
$$

We have:

$$
\begin{aligned}
b_{m}= & \left.\frac{1}{2 \pi i} \oint_{C_{\infty}}\left(\sum_{l \geq 0} \varphi_{l} k^{-l}\right)\left(\sum_{j \geq 0} \xi_{j}(x) k^{-j}\right) \partial_{x^{\prime}}^{m}\left(\sum_{i \geq 0} \xi_{i}^{\dagger}\left(x^{\prime}\right)(-k)^{-i}\right) e^{\left(x-x^{\prime}\right) z} \frac{d k}{2 \pi i}\right|_{x^{\prime}=x} \\
= & \frac{1}{2 \pi i} \oint_{C_{\infty}}\left(\sum_{l \geq 0} \varphi_{l} k^{-l}\right)\left(\sum_{j \geq 0} \xi_{j} k^{-j}\right)\left(\partial_{x}-k\right)^{m}\left(\sum_{i \geq 0} \xi_{i}(-k)^{-i}\right) \frac{d k}{2 \pi i} \\
= & \sum_{l=0}^{m}(-1)^{l} \frac{m !}{(m-l) !} \varphi_{l} \sum_{i+j+s=m-l+1}(-1)^{m-l+i+s}\left(\begin{array}{c}
m-l \\
s
\end{array}\right) \xi_{j} \partial_{x}^{s} \xi_{i}^{\dagger} .
\end{aligned}
$$

But the last sum is the coefficient of $(-1)^{m} \partial_{x}^{-m+l-1}$ in the operator $W V^{\dagger}$, so we can write:

$$
b_{m}=\sum_{l=0}^{m}(-1)^{m-l} \frac{m !}{(m-l) !} \varphi_{l}\left(W V^{\dagger}\right)_{-m+l-1}=0 \quad \text { for all } m \geq 0 .
$$

This is a homogeneous triangular system of linear equations for the coefficients $\left(W V^{\dagger}\right)_{-l}$. The unique solution is $\left(W V^{\dagger}\right)_{-l}=0$ for all $l \geq 1$, hence $W V^{\dagger}=1$, i.e. $V=\left(W^{\dagger}\right)^{-1}$. 


\subsection{Theta-functional formulae}

The Baker-Akhiezer function can be explicitly written in terms of the Riemann-theta function [12]:

$$
\begin{aligned}
\Psi_{B A}(P)= & \exp \left(x \Omega_{1}(P)+\sum_{j \geq 1} t_{j} \Omega_{j}(P)\right) \\
& \times \frac{\theta\left(\vec{A}(P)+\vec{U}_{1} x+\sum_{j \geq 1} \vec{U}_{j} t_{j}-\vec{A}(\mathcal{D})-\vec{K}\right) \theta\left(\vec{A}(\mathcal{D})+\vec{K}-\vec{A}\left(P_{\infty}\right)\right)}{\theta(\vec{A}(P)-\vec{A}(\mathcal{D})-\vec{K}) \theta\left(\vec{U}_{1} x+\sum_{j \geq 1} \vec{U}_{j} t_{j}-\vec{A}(\mathcal{D})-\vec{K}+\vec{A}\left(P_{\infty}\right)\right)} .
\end{aligned}
$$

For the proof of (3.33) it is enough to check that the right-hand side of (3.33) $(a)$ is a single-valued function on $\Gamma$, i.e. does not depend on the choice of path of integration in the definition of the Abel map and the Abelian integral $\Omega_{j}$ (which is assumed to be the same for the both objects); (b) has required exponential singularity at the marked point $P_{\infty} ;(c)$ outside of $P_{\infty}$ is meromorphic with divisor of poles at the divisor $\mathcal{D}$.

The proof of $(a)$ directly follows from the monodromy properties of the theta-function and the definition (3.8) of the vectors $U_{j}$. The proof of $(b)$ follows from the definition of the differentials $d \Omega_{j}$. The $(c)$ part follows from the Jacoby inversion theorem above.

The corresponding expression for the adjoint Baker-Akhiezer function is

$$
\begin{aligned}
\Psi_{B A}^{\dagger}(P)= & \exp \left(-x \Omega_{1}(P)-\sum_{j \geq 1} t_{j} \Omega_{j}(P)\right) \\
& \times \frac{\theta\left(\vec{A}(P)-\vec{U}_{1} x-\sum_{j \geq 1} \vec{U}_{j} t_{j}-\vec{A}\left(\mathcal{D}^{\dagger}\right)-\vec{K}\right) \theta\left(\vec{A}\left(\mathcal{D}^{\dagger}\right)+\vec{K}-\vec{A}\left(P_{\infty}\right)\right)}{\theta\left(\vec{A}(P)-\vec{A}\left(\mathcal{D}^{\dagger}\right)-\vec{K}\right) \theta\left(\vec{U}_{1} x+\sum_{j \geq 1} \vec{U}_{j} t_{j}+\vec{A}\left(\mathcal{D}^{\dagger}\right)+\vec{K}-\vec{A}\left(P_{\infty}\right)\right)}
\end{aligned}
$$

Using the relation (4.16), one can rewrite it in the form

$$
\begin{aligned}
\Psi_{B A}^{\dagger}(P) & =\exp \left(-x \Omega_{1}(P)-\sum_{j \geq 1} t_{j} \Omega_{j}(P)\right) \\
& \times \frac{\theta\left(\vec{A}(P)-\vec{U}_{1} x-\sum_{j \geq 1} \vec{U}_{j} t_{j}+\vec{A}(\mathcal{D})+\vec{K}-2 \vec{A}\left(P_{\infty}\right)\right) \theta\left(\vec{A}(\mathcal{D})+\vec{K}-\vec{A}\left(P_{\infty}\right)\right)}{\theta\left(\vec{A}(P)+\vec{A}(\mathcal{D})+\vec{K}-2 \vec{A}\left(P_{\infty}\right)\right) \theta\left(\vec{U}_{1} x+\sum_{j \geq 1} \vec{U}_{j} t_{j}-\vec{A}(\mathcal{D})-\vec{K}+\vec{A}\left(P_{\infty}\right)\right)}
\end{aligned}
$$

In order to find the solution $u_{1}=u_{1}\left(x, t_{1}, t_{2}, t_{3}, \ldots\right)$ to the KP hierarchy, one should find the coefficient $\xi_{1}$ in the expansion

$$
\log \Psi_{B A}=x k+\sum_{j \geq 1} t_{j} k^{j}+\xi_{1} k^{-1}+O\left(k^{-2}\right) .
$$

A direct calculation with the help of the explicit formula (3.33) yields

$$
\xi_{1}=x \Omega_{11}+\sum_{i \geq 1} t_{i} \Omega_{1 i}-\partial_{x} \log \theta\left(\vec{U}_{1} x+\sum_{j \geq 1} \vec{U}_{j} t_{j}+\vec{Z}\right)+\text { const }
$$


where $\vec{Z}=-\vec{A}(\mathcal{D})-\vec{K}+\vec{A}\left(P_{\infty}\right)$. Therefore,

$$
u_{1}=-\xi_{1}^{\prime}=\partial_{x}^{2} \log \theta\left(\vec{U}_{1} x+\sum_{j \geq 1} \vec{U}_{j} t_{j}+\vec{Z}\right)-\Omega_{11}
$$

\subsection{The tau-function}

Without loss of generality we can put $x=0$ for simplicity. The dependence on $x$ can be restored by the substitution $t_{1} \rightarrow t_{1}+x$.

The theta-functional formula (3.33) for the Baker-Akhiezer function and the expansion (3.10) of the Abel map near $P_{\infty}$ allows to reformulate the above presented construction of algebraic-geometrical construction in terms of the tau-functional formulation of the KP hierarchy. Namely, we have the following theorem.

Theorem 3.3 ([12, 29]) The right hand side of the equation

$$
\tau^{\mathrm{KP}}(\mathbf{t})=\exp \left(-\frac{1}{2} \sum_{i, j \geq 1} \Omega_{i j} t_{i} t_{j}\right) \theta\left(\sum_{j \geq 1} \vec{U}_{j} t_{j}+\vec{Z}\right),
$$

where the constant vector $\vec{Z}$ is parameterized through the divisor $\mathcal{D}$ as

$$
\vec{Z}=-\vec{A}(\mathcal{D})-\vec{K}+\vec{A}\left(P_{\infty}\right)
$$

is the KP tau-function.

Proof. Equation (3.10) implies that

$$
\theta\left(\sum_{j \geq 1} \vec{U}_{j}\left(t_{j} \mp \frac{1}{j} k^{-j}\right)+\vec{Z}\right)=\theta\left( \pm \vec{A}(P)+\sum_{j \geq 1} \vec{U}_{j} t_{j}+\vec{Z} \mp \vec{A}\left(P_{\infty}\right)\right)
$$

so we see that the Baker-Akhiezer functions (3.33), (3.35) are connected with the taufunction by the standard formulas [5, 6]

$$
\begin{gathered}
\Psi_{B A}=C(k) \exp \left(\sum_{j \geq 1} t_{j} k^{j}\right) \frac{\tau^{\mathrm{KP}}\left(\mathbf{t}-\left[k^{-1}\right]\right)}{\tau^{\mathrm{KP}}(\mathbf{t})}, \\
\Psi_{B A}^{\dagger}=C^{\dagger}(k) \exp \left(-\sum_{j \geq 1} t_{j} k^{j}\right) \frac{\tau^{\mathrm{KP}}\left(\mathbf{t}+\left[k^{-1}\right]\right)}{\tau^{\mathrm{KP}}(\mathbf{t})},
\end{gathered}
$$

where $C(k), C^{\dagger}(k)$ are normalization factors such that $C(k)=1+O\left(k^{-1}\right), C^{\dagger}(k)=$ $1+O\left(k^{-1}\right)$. A simple calculation shows that

$$
\begin{aligned}
\frac{\tau^{\mathrm{KP}}\left(\mathbf{t}-\left[k_{1}^{-1}\right]-\left[k_{2}^{-1}\right]\right) \tau^{\mathrm{KP}}(\mathbf{t})}{\tau^{\mathrm{KP}}\left(\mathbf{t}-\left[k_{1}^{-1}\right]\right) \tau^{\mathrm{KP}}\left(\mathbf{t}-\left[k_{2}^{-1}\right]\right)}=\exp \left(-\sum_{i, j \geq 1} \Omega_{i j} \frac{k_{1}^{-i} k_{2}^{-j}}{i j}\right) \\
\times \frac{\theta\left(\vec{A}\left(P_{1}\right)+\vec{A}\left(P_{2}\right)+\sum_{j \geq 1} \vec{U}_{j} t_{j}+\vec{Z}\right) \theta\left(\sum_{j \geq 1} \vec{U}_{j} t_{j}+\vec{Z}\right)}{\theta\left(\vec{A}\left(P_{1}\right)+\sum_{j \geq 1} \vec{U}_{j} t_{j}+\vec{Z}\right) \theta\left(\vec{A}\left(P_{2}\right)+\sum_{j \geq 1} \vec{U}_{j} t_{j}+\vec{Z}\right)} .
\end{aligned}
$$


Using (3.16), it is straightforward to check that the tau-function (3.38) satisfies the Hirota-Miwa equation (2.45) which is the generating equation for the KP hierarchy. It appears to be equivalent to the Fay identity (3.19).

It is interesting to compare equation (3.29) and the bilinear relation (1.6) for the tau-function. They coincide if

$$
\begin{gathered}
d \Omega=\frac{\tau^{\mathrm{KP}}\left(-\left[k^{-1}\right]\right) \tau^{\mathrm{KP}}\left(\left[k^{-1}\right]\right)}{\left(\tau^{\mathrm{KP}}(0)\right)^{2}} d k \\
=\frac{\left.\theta(\vec{A}(P)-\vec{A}(\mathcal{D})-\vec{K}) \theta\left(\vec{A}(P)-\vec{A}\left(\mathcal{D}^{\dagger}\right)-\vec{K}\right)\right)}{\theta^{2}\left(\vec{A}(\mathcal{D})+\vec{K}-\vec{A}\left(P_{\infty}\right)\right)} \exp \left(-\sum_{i, j \geq 1} \Omega_{i j} \frac{k^{-i-j}}{i j}\right) d k .
\end{gathered}
$$

Using (3.17), we can rewrite this as

$$
d \Omega=C \frac{\left.\theta(\vec{A}(P)-\vec{A}(\mathcal{D})-\vec{K}) \theta\left(\vec{A}(P)-\vec{A}\left(\mathcal{D}^{\dagger}\right)-\vec{K}\right)\right)}{\theta_{*}^{2}\left(\vec{A}(P)-\vec{A}\left(P_{\infty}\right)\right)} d \zeta,
$$

where the holomorphic differential $d \zeta$ is given by (3.18). Its properties (see [26]) imply that the differential in the right hand side is a well-defined meromorphic differential on $\Gamma$ with the only second order pole at $P_{\infty}$ and $2 g$ zeros at the points of the divisors $\mathcal{D}, \mathcal{D}^{\dagger}$. Therefore, it has all the properties of the differential $d \Omega$ and hence must be proportional to it. The equality (3.44) just reflects this fact.

Remark. The function $\Psi_{B A}$ and the wave function $\Psi$ introduced in section 2.2 (see (2.12) ) differ by a normalization factor depending on $k(P)$. From (3.43) it follows that

$$
\Psi_{B A}(x, \mathbf{t}, P) \Psi_{B A}^{\dagger}\left(x, \mathbf{t}^{\prime}, P\right) d \Omega=\Psi(x, \mathbf{t}, k) \Psi^{\dagger}\left(x, \mathbf{t}^{\prime}, k\right) d k .
$$

\subsection{Curves with involution: solutions to the CKP hierarchy}

Let $\Gamma$ be a smooth genus $g$ algebraic curve with involution $\iota$ having $2(n+1)>0$ fixed points. By the Riemann-Hurwitz formula $g=2 g_{0}+n$ where $g_{0}$ is the genus of the factorcurve $\Gamma_{0}=\Gamma / \iota$. It is known that on $\Gamma$ there is a basis of $a$-and $b$-cycles with canonical intersection matrix: $a_{i} \cdot a_{j}=b_{i} \cdot b_{j}=0, a_{i} \cdot b_{j}=\delta_{i j}$; and such that in this basis the action of the involution $\iota$ has the form

$$
\iota\left(a_{i}\right)=a_{i+g_{0}}, \quad \iota\left(b_{i}\right)=b_{i+g_{0}}, i=1, \ldots, g_{0},
$$

and

$$
\iota\left(a_{i}\right)=-a_{i}, \quad \iota\left(b_{i}\right)=-b_{i}, \quad i=2 g_{0}+1, \ldots, 2 g_{0}+n .
$$

Let the marked point $P_{\infty}$ on $\Gamma$ be one of the fixed points of the involution, $\iota\left(P_{\infty}\right)=P_{\infty}$ and let $z=k^{-1}$ be a local coordinate in the neighborhood of $P_{\infty}$ that is odd with respect to the involution, $\iota^{*}(k)=-k$. From the definition of the abelian differentials $d \Omega_{j}$ in subsection 3.1 it follows that

$$
d \Omega_{j}(\iota P)=(-1)^{j} d \Omega_{j}(P)
$$


and, therefore,

$$
\Omega_{j}(\iota P)=(-1)^{j} \Omega_{j}(P) .
$$

Suppose that the divisor $\mathcal{D}$ satisfies the constraint

$$
\mathcal{D}+\iota \mathcal{D}=\mathcal{K}+2 P_{\infty}
$$

Then for the Baker-Akhiezer function defined by $\Gamma, P_{\infty}$, the local coordinate $k^{-1}$ and the divisor $\mathcal{D}$ the equation

$$
\Psi_{B A}^{\dagger}\left(t_{1}, 0, t_{3}, 0, \ldots, P\right)=\Psi_{B A}\left(t_{1}, 0, t_{3}, 0, \ldots, \iota P\right)
$$

holds. The bilinear relation (3.29) takes the form

$$
\oint_{C_{\infty}} \Psi_{B A}\left(x, \mathbf{t}_{\mathrm{o}}, P\right) \Psi_{B A}\left(x, \mathbf{t}_{\mathrm{o}}^{\prime}, \iota P\right) d \Omega(P)=0
$$

for all $\mathbf{t}_{\mathrm{o}}, \mathbf{t}_{\mathrm{o}}^{\prime}$.

Using formulas (3.33), (3.35) we can write the relation (3.51) in the explicit form:

$$
\begin{aligned}
& \Psi_{B A}\left(t_{1}, 0, t_{3}, 0, \ldots, \iota P\right)=\exp \left(\sum_{j \geq 1, j \text { odd }} t_{j} \Omega_{j}(\iota P)\right) \\
& \quad \times \frac{\theta\left(\vec{A}(\iota P)+\sum_{j \geq 1, j \text { odd }} \vec{U}_{j} t_{j}-\vec{A}(\mathcal{D})-\vec{K}\right) \theta\left(\vec{A}(\mathcal{D})+\vec{K}-\vec{A}\left(P_{\infty}\right)\right)}{\theta(\vec{A}(\iota P)-\vec{A}(\mathcal{D})-\vec{K}) \theta\left(\sum_{j \geq 1, j \text { odd }} \vec{U}_{j} t_{j}-\vec{A}(\mathcal{D})-\vec{K}+\vec{A}\left(P_{\infty}\right)\right)} \\
& =\Psi_{B A}^{\dagger}\left(t_{1}, 0, t_{3}, 0, \ldots, P\right)=\exp \left(-\sum_{j \geq 1, j \text { odd }} t_{j} \Omega_{j}(P)\right) \\
& \quad \times \frac{\left.\vec{A}(P)-\sum_{j \geq 1, j \text { odd }} \overrightarrow{U_{j}} t_{j}+\vec{A}(\mathcal{D})+\vec{K}-2 \vec{A}\left(P_{\infty}\right)\right) \theta\left(\vec{A}(\mathcal{D})+\vec{K}-\vec{A}\left(P_{\infty}\right)\right)}{\theta\left(\vec{A}(P)+\vec{A}(\mathcal{D})+\vec{K}-2 \vec{A}\left(P_{\infty}\right)\right) \theta\left(\sum_{j \geq 1, j \text { odd }} \vec{U}_{j} t_{j}-\vec{A}(\mathcal{D})-\vec{K}+\vec{A}\left(P_{\infty}\right)\right)} .
\end{aligned}
$$

The tau-function of the CKP hierarchy is the square root of

$$
\tau^{\mathrm{KP}}\left(\mathbf{t}_{\mathrm{o}}\right)=\exp \left(-\frac{1}{2} \sum_{i, j \geq 1, i, j \text { odd }} \Omega_{i j} t_{i} t_{j}\right) \theta\left(\sum_{j \geq 1, j \text { odd }} \vec{U}_{j} t_{j}-\vec{A}(\mathcal{D})-\vec{K}+\vec{A}\left(P_{\infty}\right)\right),
$$

where the divisor $\mathcal{D}$ satisfies the condition (3.50).

The statement of the following theorem is in fact a corollary of Theorem 2.1 and the above identification of the square root of (3.54) with the tau-function of the CKP hierarchy but below we give its closed algebraic-geometrical proof.

Theorem 3.4 Let $\Gamma$ be a genus $g$ smooth curve with holomorphic involution $\iota$ having at least one fixed point $P_{\infty}$ and let $Y$ be the locus in the Jacobian

$$
Y \subset J a c(\Gamma)=\left\{\vec{Z} \in Y \mid \vec{Z}+\iota(\vec{Z})=-2 \vec{A}\left(P_{\infty}\right)\right\}
$$


Then for any point $Q \in \Gamma$ and $\vec{Z} \in Y$ the equation

$$
\begin{aligned}
\theta(\vec{Z}) \partial_{1} \theta(\vec{A}(Q)-\vec{A}(\iota Q)+\vec{Z})-\theta(\vec{A}(Q)-\vec{A}(\iota Q)+\vec{Z}) \partial_{1} \theta(\vec{Z}) \\
\quad+2 \Omega_{1}(Q) \theta(\vec{Z}) \theta(\vec{A}(Q)-\vec{A}(\iota Q)+\vec{Z})=C(Q) \theta^{2}(\vec{A}(Q)+\vec{Z})
\end{aligned}
$$

with

$$
\partial_{1} \theta(\vec{Z}):=\left.\partial_{t} \theta\left(\vec{Z}+\vec{U}_{1} t\right)\right|_{t=0}
$$

holds.

Remark. Note that $Y$ is the locus of vectors such that $\vec{Z}=-\vec{A}(\mathcal{D})-\vec{K}$, where the divisor $\mathcal{D}$ satisfies the condition (3.50).

Proof. Let us fix a point $Q \in \Gamma$, an effective divisor $\mathcal{D}$ of degree $g$ and define the auxiliary Baker-Akhiezer function $\Psi_{Q}\left(\mathbf{t}_{\mathrm{o}}, P\right)$ by the following properties:

$1^{0}$. Outside $P_{\infty}$ the singularities of $\Psi_{Q}$ are poles at the divisor $\mathcal{D}+\iota Q$;

$2^{0}$. It has simple zero at the point $Q$, i.e., $\Psi_{Q}\left(\mathbf{t}_{\mathrm{o}}, Q\right)=0$;

$3^{0}$. In a small neighborhood of $P_{\infty}$ the function $\Psi_{Q}$ has the form

$$
\Psi_{Q}\left(\mathbf{t}_{\mathrm{o}}, P\right)=e^{\zeta\left(\mathbf{t}_{\mathrm{o}}, k\right)}\left(1+\sum_{j \geq 1} \xi_{j, Q}\left(\mathbf{t}_{\mathrm{o}}\right) k^{-j}\right), \quad k=k(P)
$$

The standard argument shows that this function is unique up to a common factor. The explicit formula for $\Psi_{Q}$ in theta-functions is

$$
\Psi_{Q}\left(\mathbf{t}_{\mathrm{o}}, P\right)=\frac{\theta\left(\vec{A}(P)-\vec{A}(\iota Q)+\vec{A}(Q)+\vec{Z}_{\mathbf{t}_{\mathrm{o}}}\right) \theta(\vec{Z})}{\theta\left(\vec{A}(Q)-\vec{A}(\iota Q)+\vec{Z}_{\mathbf{t}_{\mathrm{o}}}\right) \theta(\vec{A}(P)+\vec{Z})} \exp \left(\Omega_{0}(P)+\sum_{j \geq 1, \text { odd }} t_{j} \Omega_{j}(P)\right)
$$

where $\vec{Z}_{\mathbf{t}_{\mathrm{o}}}=\vec{Z}+\sum_{j \geq 1, \text { odd }} U_{j} t_{j}$ and $\Omega_{0}$ is the abelian integral of the normalized dipole differential $d \Omega_{0}$ with simple poles at the points $Q, \iota Q$ with residues \pm 1 :

$$
\Omega_{0}(P)=\int_{Q_{0}}^{P} d \Omega_{0}
$$

Remark. The standard Baker-Akhiezer function $\Psi_{B A}$ corresponds to the case $Q=P_{\infty}$.

Consider the differential $\widetilde{d \Omega}(P)=\partial_{t_{1}} \Psi_{Q}(P) \Psi_{Q}(\iota P) d \Omega(P)$, where $d \Omega$ is the differential entering the bilinear relation (3.29). It is a meromorphic differential on $\Gamma$ with the only pole at $P_{\infty}$. Hence it has no residue $P_{\infty}$. Computing the residue in terms of the coefficients of the expansion (3.57), we get

$$
2 \xi_{2, Q}-\xi_{1, Q}^{2}+\partial_{t_{1}} \xi_{1, Q}+c_{1}=0
$$

where $c_{1}$ is a constant defined by the Laurent expansion of $d \Omega$ at $P_{\infty}$. 
Consider now the differential $d \Omega_{Q}(P)=\Psi_{Q}(P) \Psi_{B A}(\iota P) d \Omega(P)$. It is a meromorphic differential with poles at $P_{\infty}$ and $\iota Q$. Therefore,

$$
f_{Q}:=\underset{P_{\infty}}{\operatorname{res}} d \Omega_{Q}=\xi_{1, Q}-\xi_{1}=-\underset{\iota Q}{\operatorname{res}} d \Omega_{Q}=-\phi_{Q} \phi,
$$

where

$$
\phi_{Q}:=\operatorname{res}_{\iota Q}\left(\Psi_{Q} d \Omega\right), \quad \phi=\Psi_{B A}\left(\mathbf{t}_{\mathrm{o}}, \iota Q\right) .
$$

The residue argument for the differential $\widetilde{d \Omega}_{Q}(P)=\partial_{t_{1}} \Psi_{Q}(P) \Psi_{B A}(\iota P) d \Omega(P)$ gives the relation

$$
\xi_{2, Q}+\xi_{2}-\xi_{1, Q} \xi_{1}+\partial_{t_{1}} \xi_{1, Q}+c_{1}=-\left(\partial_{t_{1}} \phi_{Q}\right) \phi .
$$

Then, using (3.59), we obtain

$$
\frac{1}{2}\left(f_{Q}^{2}+\partial_{t_{1}} f_{Q}\right)=-\left(\partial_{t_{1}} \phi_{Q}\right) \phi
$$

From comparison of (3.60) and (3.63) it follows that

$$
\partial_{t_{1}} \log \phi_{Q}=\frac{1}{2}\left(f_{Q}+\partial_{t_{1}} \log f_{Q}\right)
$$

Recalling the definition of $\phi_{Q}$ and using formula (3.58), we get

$$
\partial_{t_{1}} \log \phi_{Q}=\partial_{t_{1}} \log \left(\frac{\theta\left(\vec{A}(Q)+\vec{Z}_{\mathbf{t}_{\mathrm{o}}}\right)}{\theta\left(\vec{A}(Q)-\vec{A}(\iota Q)+\vec{Z}_{\mathbf{t}_{\mathrm{o}}}\right)}\right)+\Omega_{1}(\iota Q) .
$$

The expansion of (3.58) around $P_{\infty}$ yields

$$
f_{Q}=\partial_{t_{1}} \log \left(\frac{\theta\left(\vec{Z}_{\mathbf{t}_{\circ}}\right)}{\theta\left(\vec{A}(Q)-\vec{A}(\iota Q)+\vec{Z}_{\mathbf{t}_{\mathrm{o}}}\right)}\right)+\Omega_{01},
$$

where $\Omega_{01}$ equals the coefficient at $k^{-1}$ in the expansion of $\Omega_{0}$ at $P_{\infty}$. The Riemann's bilinear relation for the differentials $d \Omega_{1}$ and $d \Omega_{0}$ has the form

$$
\Omega_{01}=\Omega_{1}(\iota Q)-\Omega_{1}(Q)=2 \Omega_{1}(\iota Q) .
$$

Therefore, equations (3.63) and (3.66) imply

$$
\partial_{t_{1}} \log \left(\frac{\theta^{2}\left(\vec{A}(Q)+\vec{Z}_{\mathbf{t}_{\circ}}\right)}{\theta\left(\vec{A}(Q)-\vec{A}(\iota Q)+\vec{Z}_{\mathbf{t}_{\circ}}\right) \theta\left(\vec{Z}_{\mathbf{t}_{\circ}}\right)}\right)=\partial_{t_{1}} \log f_{Q} .
$$

Equation (3.66) and (3.68) with $\mathbf{t}_{\mathrm{o}}=0$ after integration in $t_{1}$ give (3.56) with constant $C(Q, \vec{Z})$ which is $\partial_{t_{1}}$-invariant, i.e. $C(Q, \vec{Z})=C\left(Q, \vec{Z}+t_{1} \vec{U}_{1}\right)$ for any value of $t_{1}$. For a generic curve the complex line $\vec{Z}+t_{1} \vec{U}_{1}$ is dense in the Jacobian. Hence, the integration constant $C$ does not depend on $\vec{Z}$ and depends on $Q$ only. Since the matrix of $b$-periods depends analytically on the curve and $C$ is independent of $\vec{Z}$ for generic curve it is independent of $\vec{Z}$ for any curve. 


\subsection{Degeneration of algebraic-geometrical solutions: soliton so- lutions}

The algebraic-geometrical integration scheme naturally extends to the case of singular curves. In particular, the case when $\Gamma$ is the Riemann sphere $\mathbb{C} P^{1}$ with nodes (double points) corresponds to soliton solutions. $N$-soliton solutions of the CKP hierarchy are obtained by imposing certain constraints on the parameters of $2 N$-soliton solutions to the KP hierarchy. We recall that $\tau=\sqrt{\tau^{\mathrm{KP}}}$, with "even" times $t_{2 k}$ put equal to zero and it is implied that the parameters of the KP tau-function $\tau^{\mathrm{KP}}$ are chosen in a special way.

$M$-solutions of the KP hierarchy are constructed starting from a singular curve which is $\mathbb{C} P^{1}$ with $M$ double points. Let $z$ be the global coordinate. The Baker-Akhiezer function has simple poles at $M$ points $q_{i}$. It has the form

$$
\Psi^{\mathrm{KP}}(\mathbf{t}, z)=\exp \left(\sum_{j \geq 1} t_{j} z^{j}\right)\left(1+\sum_{l=1}^{M} \frac{y_{l}(\mathbf{t})}{z-q_{l}}\right) .
$$

Let us impose $M$ linear conditions of the form

$$
\underset{z=q_{i}}{\operatorname{res}}\left[\Psi^{\mathrm{KP}}(\mathbf{t}, z) d z\right]=-\alpha_{i}\left(p_{i}-q_{i}\right) \Psi^{\mathrm{KP}}\left(\mathbf{t}, p_{i}\right), \quad i=1, \ldots, M,
$$

which mean that the points $p_{i}, q_{i}$ are glued together forming a double point. Here $\alpha_{i}$ are complex parameters. These conditions make the Baker-Akhiezer function unique (up to a common multiplier). The conditions (3.70) are equivalent to the following linear system for $y_{l}$ :

$$
y_{i}+\sum_{l=1}^{M} \frac{\tilde{\alpha}_{i} y_{l}}{p_{i}-q_{l}}=-\tilde{\alpha}_{i}
$$

where

$$
\tilde{\alpha}_{i}=\alpha_{i}\left(p_{i}-q_{i}\right) \exp \left(\sum_{j \geq 1}\left(p_{i}^{j}-q_{i}^{j}\right) t_{j}\right) .
$$

Solving this system, we obtain the Baker-Akhiezer function in the explicit form:

$$
\Psi^{\mathrm{KP}}=\frac{\left|\begin{array}{ccccc}
1 & \frac{1}{z-q_{1}} & \frac{1}{z-q_{2}} & \ldots & \frac{1}{z-q_{M}} \\
\tilde{\alpha}_{1} & 1+\frac{\tilde{\alpha}_{1}}{p_{1}-q_{1}} & \frac{\tilde{\alpha}_{1}}{p_{1}-q_{2}} & \ldots & \frac{\tilde{\alpha}_{1}}{p_{1}-q_{M}} \\
\tilde{\alpha}_{2} & \frac{\tilde{\alpha}_{2}}{p_{2}-q_{1}} & 1+\frac{\tilde{\alpha}_{2}}{p_{2}-q_{2}} & \ldots & \frac{\tilde{\alpha}_{2}}{p_{2}-q_{M}} \\
\ldots & \cdots & \cdots & \ldots & \cdots \\
\tilde{\alpha}_{M} & \frac{\tilde{\alpha}_{M}}{p_{M}-q_{1}} & \frac{\tilde{\alpha}_{M}}{p_{M}-q_{2}} & \ldots & 1+\frac{\tilde{\alpha}_{M}}{p_{M}-q_{M}}
\end{array}\right|}{\left|\begin{array}{cccc}
1+\frac{\tilde{\alpha}_{1}}{p_{1}-q_{1}} & \frac{\tilde{\alpha}_{1}}{p_{1}-q_{2}} & \ldots & \frac{\tilde{\alpha}_{1}}{p_{1}-q_{M}} \\
\frac{\tilde{\alpha}_{2}}{p_{2}-q_{1}} & 1+\frac{\tilde{\alpha}_{2}}{p_{2}-q_{2}} & \ldots & \frac{\tilde{\alpha}_{2}}{p_{2}-q_{M}} \\
\cdots & \cdots & \ldots & \cdots \\
\frac{\tilde{\alpha}_{M}}{p_{M}-q_{1}} & \frac{\tilde{\alpha}_{M}}{p_{M}-q_{2}} & \ldots & 1+\frac{\tilde{\alpha}_{M}}{p_{M}-q_{M}}
\end{array}\right|} \exp \left(\sum_{j \geq 1} z^{j}\right) .
$$

The denominator of this expression is the tau-function. 
The general KP tau-function for $M$-soliton solution has $3 M$ arbitrary parameters $\alpha_{i}$, $p_{i}, q_{i}(i=1, \ldots, M)$ and is given by

$$
\tau^{\mathrm{KP}}(x, \mathbf{t})=\operatorname{det}_{1 \leq i, j \leq M}\left(\delta_{i j}+\alpha_{i} \frac{p_{i}-q_{i}}{p_{i}-q_{j}} \exp \left(\left(p_{i}-q_{i}\right) x+\sum_{k \geq 1}\left(p_{i}^{k}-q_{i}^{k}\right) t_{k}\right)\right) .
$$

Let us denote this tau-function as

$$
\tau^{\mathrm{KP}}\left[\begin{array}{c}
\alpha_{1} \\
p_{1}, q_{1}
\end{array} ; \begin{array}{c}
\alpha_{2}, q_{2} \\
p_{2}
\end{array} ; \begin{array}{c}
\alpha_{3} \\
p_{3}, q_{3}
\end{array} ; \begin{array}{c}
\alpha_{4} \\
p_{4}, q_{4}
\end{array} ; \cdots ; \begin{array}{c}
\alpha_{2 N-1} \\
p_{M-1}, q_{M-1}
\end{array} ; \begin{array}{c}
\alpha_{2 N} \\
p_{M}, q_{M}
\end{array}\right] .
$$

The parameters $p_{i}, q_{i}$ are sometimes called momenta of solitons.

In the CKP case we have the involution $z \rightarrow-z$ which means that the double points should be symmetric under the involution. The multi-soliton tau-function of the CKP hierarchy is the square root of the $\tau^{\mathrm{KP}}$ specialized as

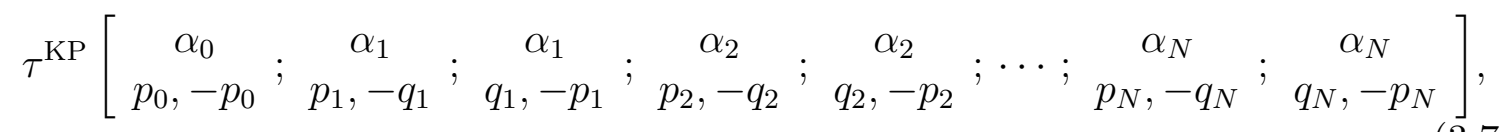

where it is assumed that even times evolution is suppressed $\left(t_{2 k}=0\right.$ for all $\left.k \geq 1\right)$. Clearly, the total number of independent parameters is $3 N+2$. If $\alpha_{0}=0$, the tau-function (3.74) reduces to

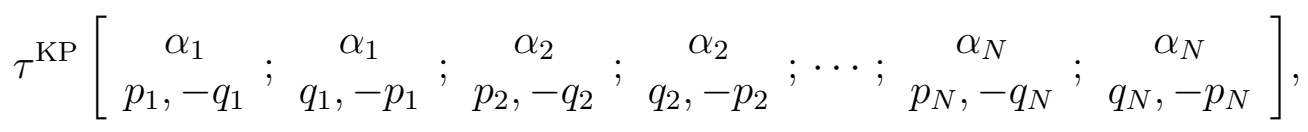

and it is this tau-function which is usually called the $N$-soliton CKP tau-function in the literature (see, e.g. [7]). It is a specialization of $2 N$-soliton KP tau-function and has $3 N$ free parameters.

The simplest example is one-soliton solution. The tau-function for one CKP soliton is the square root of a specialization of 2-soliton tau-function of the KP hierarchy:

$$
\tau^{\mathrm{KP}}=1+2 \alpha w-\frac{\alpha^{2}(p-q)^{2}}{4 p q} w^{2}
$$

where

$$
w=e^{(p+q) x+\zeta\left(\mathbf{t}_{\mathrm{o}}, p\right)+\zeta\left(\mathbf{t}_{\mathrm{o}}, q\right)}, \quad \zeta\left(\mathbf{t}_{\mathrm{o}}, z\right) \text { is given by (2.13)} .
$$

A direct calculation shows that $\partial_{x} \psi^{2}$ (where $\psi$ is given by (2.17)) for the solution (3.76) is a full square for all $z$.

Remark. It is instructive to prove directly that the tau-functions (3.74) and (3.75) satisfy equation (2.48). Consider (3.75) first. We represent the tau-function as

$$
\tau^{\mathrm{KP}}=\operatorname{det}_{2 N \times 2 N}(I+H K)
$$

where $H$ is the diagonal matrix $W_{j k}=\delta_{j k} W_{j}$ with matrix elements

$$
H_{2 i-1}=\alpha_{i}\left(p_{i}+q_{i}\right) \exp \left(\left(p_{i}+q_{i}\right) x+\sum_{k \geq 1} t_{k}\left(p_{i}^{k}-\left(-q_{i}\right)^{k}\right)\right), \quad i=1, \ldots, N,
$$




$$
H_{2 i}=\alpha_{i}\left(p_{i}+q_{i}\right) \exp \left(\left(p_{i}+q_{i}\right) x+\sum_{k \geq 1} t_{k}\left(q_{i}^{k}-\left(-p_{i}\right)^{k}\right)\right), \quad i=1, \ldots, N,
$$

and $K$ is the Cauchy matrix $K_{j k}=1 /\left(x_{j}-y_{k}\right)$ with $x_{2 i-1}=-y_{2 i}=p_{i}, x_{2 i}=-y_{2 i-1}=q_{i}$, $i=1, \ldots, N$. We have:

$$
\begin{gathered}
\left.\partial_{t_{2 m}} \log \tau^{\mathrm{KP}}\right|_{t_{2 k}=0}=\left.\partial_{t_{2 m}} \log \operatorname{det}(I+H K)\right|_{t_{2 k}=0}=\left.\partial_{t_{2 m}} \operatorname{tr} \log (I+H K)\right|_{t_{2 k}=0} \\
=\operatorname{tr}\left[V H K(I+H K)^{-1}\right]=\operatorname{tr} V-\operatorname{tr}\left[V(I+H K)^{-1}\right]
\end{gathered}
$$

where $V$ is the diagonal matrix $V_{j k}=\delta_{j k} V_{j}$ with the matrix elements

$$
V_{2 i-1}=-V_{2 i}=p_{i}^{2 m}-q_{i}^{2 m} \text {. }
$$

Note also that when all even times are put equal to zero, we have also $H_{2 i-1}=-H_{2 i}$. Obviously, $\operatorname{tr} V=0$. A careful inspection shows that $(I+H K)_{2 i-1,2 i-1}^{-1}=(I+H K)_{2 i, 2 i}^{-1}$,

and, therefore, $\operatorname{tr}\left[V(I+H K)^{-1}\right]=0$, too, and the conditions (2.48) are satisfied. Indeed, permuting rows and columns, one can see that the diagonal $(2 i-1,2 i-1)$ and $(2 i, 2 i)$ minors of the matrix $I+H K$ are equal. As for the tau-function (3.74) with $\alpha_{0} \neq 0$, it is obvious that the additional pair of soliton momenta of the form $p_{0},-p_{0}$ does not lead to any extra dependence on the even times, and so the conditions (2.48) are still satisfied.

\section{$4 \quad$ Elliptic solutions}

By elliptic solutions of the CKP equation (2.10) we mean solutions $u$ that are doubleperiodic in the complex plane of the variable $x$ with periods $2 \omega_{1}, 2 \omega_{2}, \operatorname{Im}\left(\omega_{2} / \omega_{1}\right)>0$. Equations of motion for their poles and their algebraic integrability is an easy corollary of the established above relation between the CKP and KP hierarchies and the welldeveloped theory of elliptic solutions to the KP hierarchy, equivalent to the theory of the elliptic Calogero-Moser (eCM) system.

Namely, elliptic solutions of the CKP equation can be extended to elliptic solutions of the KP equation and further to the whole KP hierarchy. From that perspective the pole dynamics of the elliptic solutions of the CKP equation in $t_{3}$ is just the restriction of $t_{3}$-dynamics generated by the Hamiltonian $H_{3}$ of the eCM system,

$$
\left\{\begin{array}{l}
\dot{x}_{i}=-3 p_{i}^{2}+3 \sum_{j \neq i} \wp\left(x_{i}-x_{j}\right)-6 c \\
\dot{p}_{i}=-3 \sum_{j \neq i}\left(p_{i}+p_{j}\right) \wp^{\prime}\left(x_{i}-x_{j}\right),
\end{array}\right.
$$

onto the locus of turning points $p_{i}=0$ that is invariant under $\mathbf{t}_{\mathrm{o}}$ flows of the eCM system, i.e.

$$
\dot{x}_{i}=3 \sum_{k \neq i} \wp\left(x_{i}-x_{k}\right)-6 c,
$$

where $c$ is a constant and dot means the $t_{3}$-derivative. Here $\wp$ is the Weiershtrass $\wp$ function which is an even double-periodic function with periods $2 \omega_{1}, 2 \omega_{2}$ having second 
order poles at the lattice points $2 \omega_{1} m_{1}+2 \omega_{2} m_{2}$ with integer $m_{1}, m_{2}$ and

$$
\wp(x)=\frac{1}{x^{2}}+O\left(x^{2}\right), \quad x \rightarrow 0
$$

For further use recall the definitions of the Weierstrass functions. The Weierstrass $\sigma$-function is given by the infinite product

$$
\sigma(x)=\sigma\left(x \mid \omega_{1}, \omega_{2}\right)=x \prod_{s \neq 0}\left(1-\frac{x}{s}\right) e^{\frac{x}{s}+\frac{x^{2}}{2 s^{2}}}, \quad s=2 \omega_{1} m_{1}+2 \omega_{2} m_{2}, \quad m_{1}, m_{2} \in \mathbb{Z} .
$$

The Weierstrass $\zeta$ - and $\wp$-functions are connected with the $\sigma$-function as follows: $\zeta(x)=$ $\sigma^{\prime}(x) / \sigma(x), \wp(x)=-\zeta^{\prime}(x)=-\partial_{x}^{2} \log \sigma(x)$.

The algebraic integrability of the eCM system established in [20] restricted to the locus of turning points can be stated as follows.

Theorem 4.1 For each set of constants $x_{i}^{0} \neq x_{j}^{0}$ define the algebraic curve $\Gamma$ by the characteristic equation $\operatorname{det}(z I-L)=0$ for the matrix

$$
L_{i i}=0, \quad L_{i j}=-\Phi\left(x_{i}^{0}-x_{j}^{0}, \lambda\right), \quad i \neq j,
$$

where

$$
\Phi(x, \lambda)=\frac{\sigma(x+\lambda)}{\sigma(\lambda) \sigma(x)} e^{-\zeta(\lambda) x} .
$$

Let $P_{\infty}$ be the point on $\Gamma$ that is the pre-image of $\lambda=0$ in the neighborhood of which $z$ has the expansion $z=-(n-1) \lambda^{-1}+O(\lambda)$. Then the solution of (4.2) with the initial conditions $x_{i}(0)=x_{i}^{0}$ are roots $x_{i}\left(t_{3}\right)$ of the equation

$$
\theta\left(\vec{U}_{1} x_{i}+\vec{U}_{3} t_{3}+\vec{Z} \mid T\right)=0
$$

Here $\theta(z \mid T)$ is Riemann theta-function defined by the matrix of b-periods of normalized holomorphic differentials on $\Gamma$; the vectors $\vec{U}_{j}$ are given by (3.8) with $d \Omega_{j}$ defined in (3.6); the vector $\vec{Z}$ is in the locus $Y$ defined in (3.55), where the involution $\iota$ of the Jacobian is induced by the involution $\iota(z, \lambda) \rightarrow(-z,-\lambda)$ of $\Gamma$.

The elliptic solutions are particular cases of the general algebraic-geometrical solutions considered in the previous section. The corresponding spectral data are singled out by the following constraint: the vectors $2 \omega_{1} \vec{U}_{1}, 2 \omega_{2} \vec{U}_{1}$ are in the lattice of periods of the Jacobian of the spectral curve, where $\vec{U}_{1}$ is the vector of $b$-periods of the normalized differential with the only pole (of order 2 ) at the marked point $P_{\infty}$.

\subsection{The generating problem}

For completeness, in this section we present the scheme proposed in [20] which allows one to derive the equations of motion for poles of elliptic solutions to a variety of soliton equations together with their Lax-type representation (see more in [21]). With the help of this scheme we will get the equations (4.2) and the Lax matrix (4.3) directly without use of relations to the theory of the eCM system. 
The elliptic solution of the CKP equation is an elliptic function with double poles at the points $x_{i}$ :

$$
u=-\frac{1}{2} \sum_{i=1}^{n} \wp\left(x-x_{i}\right)+c,
$$

where $c$ is a constant. The poles depend on the times $t_{3}, t_{5}$ (as well as on the higher times) and are assumed to be all distinct. The corresponding CKP tau-function has the form

$$
\tau\left(x, \mathbf{t}_{\mathrm{o}}\right)=C_{0} e^{c x^{2} / 2}\left(\prod_{i=1}^{n} \sigma\left(x-x_{i}\left(\mathbf{t}_{\mathrm{o}}\right)\right)\right)^{1 / 2} .
$$

In the rest of this section we denote $t_{3}=t$. According to the scheme proposed in [20], the basic tool is the auxiliary linear problem $\partial_{t} \Psi=B_{3} \Psi$ for the wave function $\Psi$, i.e.,

$$
\partial_{t} \Psi=\partial_{x}^{3} \Psi+6 u \partial_{x} \Psi+3 u^{\prime} \Psi
$$

for which one can state the following problem: characterize an elliptic in $x$ function $u$ of the form (4.6) for which equation (4.8) has double-Bloch solutions $\Psi(x)$, i.e., solutions such that $\Psi\left(x+2 \omega_{\alpha}\right)=B_{\alpha} \Psi(x)$ with some Bloch multipliers $B_{\alpha}$. Equations (2.17), (2.16) imply that the wave function has simple poles at the points $x_{i}$. Therefore, if a double-Bloch solution exists, then it is of the following pole ansatz form:

$$
\Psi=e^{x z+t z^{3}} \sum_{i=1}^{n} c_{i} \Phi\left(x-x_{i}, \lambda\right)
$$

where the coefficients $c_{i}$ do not depend on $x$ (but do depend on $t, z$ and $\lambda$ ). Indeed, the function $\Phi(x, \lambda)$ given by formula (4.4) has the following monodromy properties:

$$
\Phi\left(x+2 \omega_{\alpha}, \lambda\right)=e^{2\left(\zeta\left(\omega_{\alpha}\right) \lambda-\zeta(\lambda) \omega_{\alpha}\right)} \Phi(x, \lambda), \quad \alpha=1,2 .
$$

Therefore, the wave function $\Psi$ given by (4.9) is a double-Bloch function with Bloch multipliers $B_{\alpha}=e^{2\left(\omega_{\alpha} z+\zeta\left(\omega_{\alpha}\right) \lambda-\zeta(\lambda) \omega_{\alpha}\right)}$ parameterized by $z$ and $\lambda$.

In what follows we will often suppress the second argument of $\Phi$ writing simply $\Phi(x)=\Phi(x, \lambda)$. For further use note also that $\Phi$ has a simple pole at $x=0$ with residue 1. The coefficients $\beta_{1}, \beta_{2}$ of its expansion

$$
\Phi(x, \lambda)=\frac{1}{x}+\beta_{1} x+\beta_{2} x^{2}+O\left(x^{3}\right) \quad \text { as } x \rightarrow 0,
$$

are equal to

$$
\beta_{1}=-\frac{1}{2} \wp(\lambda), \quad \beta_{2}=-\frac{1}{6} \wp^{\prime}(\lambda)
$$

The function $\Phi$ We will also need the $x$-derivatives $\Phi^{\prime}(x, \lambda)=\partial_{x} \Phi(x, \lambda), \Phi^{\prime \prime}(x, \lambda)=$ $\partial_{x}^{2} \Phi(x, \lambda)$ and so on.

Theorem 4.2 The equations of motion (4.2) for poles $x_{i}$ of elliptic solutions as functions of $t=t_{3}$ have the following commutation representation of the Manakov's triple kind:

$$
\dot{L}+[L, M]=3 D^{\prime}(z I-L)
$$

where

$$
L_{i i}=0, \quad L_{i j}=-\Phi\left(x_{i}-x_{j}, \lambda\right), \quad i \neq j ;
$$

the matrix $M$ is defined by (4.18), and $D^{\prime}$ is the diagonal matrix $D_{i k}^{\prime}=\delta_{i k} \sum_{j \neq i} \wp^{\prime}\left(x_{i}-x_{j}\right)$. 
Proof. Substituting (4.9) into (4.8) with $u=-\frac{1}{2} \sum_{i} \wp\left(x-x_{i}\right)+c$, we get:

$$
\begin{aligned}
\sum_{i} \dot{c}_{i} \Phi\left(x-x_{i}\right)-\sum_{i} c_{i} \dot{x}_{i} \Phi^{\prime}\left(x-x_{i}\right)=3 z^{2} \sum_{i} c_{i} \Phi^{\prime}\left(x-x_{i}\right)+3 z \sum_{i} c_{i} \Phi^{\prime \prime}\left(x-x_{i}\right)+\sum_{i} c_{i} \Phi^{\prime \prime \prime}\left(x-x_{i}\right) \\
-3 z\left(\sum_{k} \wp\left(x-x_{k}\right)\right)\left(\sum_{i} c_{i} \Phi\left(x-x_{i}\right)\right)-3\left(\sum_{k} \wp\left(x-x_{k}\right)\right)\left(\sum_{i} c_{i} \Phi^{\prime}\left(x-x_{i}\right)\right) \\
-\frac{3}{2}\left(\sum_{k} \wp^{\prime}\left(x-x_{k}\right)\right)\left(\sum_{i} c_{i} \Phi\left(x-x_{i}\right)\right)+6 c z \sum_{i} c_{i} \Phi\left(x-x_{i}\right)+6 c \sum_{i} c_{i} \Phi^{\prime}\left(x-x_{i}\right) .
\end{aligned}
$$

It is enough to cancel all poles in the fundamental domain which are at the points $x_{i}$ (up to fourth order). It is easy to see that poles of the fourth order cancel identically. A direct calculation shows that the conditions of cancellation of third, second and first order poles have the form

$$
\begin{gathered}
z c_{i}=-\sum_{k \neq i} c_{k} \Phi\left(x_{i}-x_{k}\right), \\
c_{i} \dot{x}_{i}=-3 z^{2} c_{i}+3 c_{i} \sum_{k \neq i} \wp\left(x_{i}-x_{k}\right)-3 z \sum_{k \neq i} c_{k} \Phi\left(x_{i}-x_{k}\right)-6 c c_{i}, \\
\dot{c}_{i}=-3\left(\beta_{1} z+\beta_{2}\right) c_{i}-3 z c_{i} \sum_{k \neq i} \wp\left(x_{i}-x_{k}\right)+\frac{3}{2} c_{i} \sum_{k \neq i} \wp^{\prime}\left(x_{i}-x_{k}\right) \\
-3 z \sum_{k \neq i} c_{k} \Phi^{\prime}\left(x_{i}-x_{k}\right)-\frac{3}{2} \sum_{k \neq i} c_{k} \Phi^{\prime \prime}\left(x_{i}-x_{k}\right)+6 c z c_{i}
\end{gathered}
$$

which have to be valid for all $i=1, \ldots, n$. Substitution of (4.14) into (4.15) gives (4.2) (if the coefficients $c_{i}$ are not identically zero). The conditions (4.14), (4.16) can be rewritten in the matrix form as linear problems for a vector $\mathbf{c}=\left(c_{1}, \ldots, c_{n}\right)^{T}$ :

$$
\left\{\begin{array}{l}
L \mathbf{c}=z \mathbf{c} \\
\dot{\mathbf{c}}=M \mathbf{c}
\end{array}\right.
$$

where $L$ is the matrix (4.13),

$$
M=-3\left(\beta_{1} z+\beta_{2}-2 c z\right) I-3 z B-3 z D-\frac{3}{2} C+\frac{3}{2} D^{\prime}
$$

and the $n \times n$ matrices $I, B, C, D$, are given by $I_{i k}=\delta_{i k}$,

$$
\begin{aligned}
& B_{i k}=\left(1-\delta_{i k}\right) \Phi^{\prime}\left(x_{i}-x_{k}\right), \\
& C_{i k}=\left(1-\delta_{i k}\right) \Phi^{\prime \prime}\left(x_{i}-x_{k}\right), \\
& D_{i k}=\delta_{i k} \sum_{j \neq i} \wp\left(x_{i}-x_{j}\right),
\end{aligned}
$$

The matrices $L, B, C$ are off-diagonal while the matrices $D, D^{\prime}$ are diagonal.

The linear system (4.17) is overdetermined. Differentiating the first equation in (4.17) with respect to $t$, we see that the compatibility condition of the linear problems (4.17) is

$$
(\dot{L}+[L, M]) \mathbf{c}=0 .
$$


One can prove the following matrix identity (see the appendix):

$$
\dot{L}+[L, M]=3 D^{\prime}(z I-L)-[\dot{X}-3 D, B]
$$

where $X$ is the diagonal matrix $X_{i k}=\delta_{i k} x_{i}$. Since $(z I-L) \mathbf{c}=0$ according to (4.14) and $\dot{X}=3 D-6 c I$ according to (4.17), we see from (4.21) that the compatibility condition (4.20) is satisfied. From (4.21) it follows that the equations of motion have the commutation representation of the Manakov's triple kind (4.12) [30].

\subsection{The integrals of motion and the spectral curve}

It follows from equation (4.12) that the characteristic polynomial of the matrix $L$ is an integral of motion. Indeed,

$$
\begin{gathered}
\frac{d}{d t} \log \operatorname{det}(L-z I)=\frac{d}{d t} \operatorname{tr} \log (L-z I) \\
=\operatorname{tr}\left[\dot{L}(L-z I)^{-1}\right]=-3 \operatorname{tr} D^{\prime}=0,
\end{gathered}
$$

where we have used equation (4.12) and the fact that $\operatorname{tr} D^{\prime}=\sum_{i \neq j} \wp^{\prime}\left(x_{i}-x_{j}\right)=0\left(\wp^{\prime}\right.$ is an odd function). The expression $R(z, \lambda)=\operatorname{det}(z I-L(\lambda))$ is a polynomial in $z$ of degree $n$. Its coefficients are integrals of motion (some of them may be trivial). For example:

$$
\begin{array}{ll}
n=2: & \operatorname{det}_{2 \times 2}(z I-L)=z^{2}+\wp\left(x_{12}\right)-\wp(\lambda), \\
n=3: & \operatorname{det}_{3 \times 3}(z I-L)=z^{3}+z\left(\wp\left(x_{12}\right)+\wp\left(x_{13}\right)+\wp\left(x_{23}\right)-3 \wp(\lambda)\right)-\wp^{\prime}(\lambda),
\end{array}
$$

where $x_{i k} \equiv x_{i}-x_{k}$.

Remark. Although the Lax equation for matrices $L, M$ does not hold, it follows from (4.22) that traces of the Lax matrix $L$ (and therefore its eigenvalues) are integrals of motion: $\partial_{t} \operatorname{tr} L^{m}=0, m \geq 1$. (This is equivalent to the equalities $\operatorname{tr}\left(D^{\prime} L^{m}\right)=0$ for $m \geq 1$ which are based on certain non-trivial identities for the $\wp$-function.) This mans that the time evolution is an isospectral transformation of the Lax matrix $L$. Therefore, there should exist a matrix $M_{0}$ such that the Lax equation $\dot{L}+\left[L, M_{0}\right]=0$ holds. In order to find it explicitly, we first note that by virtue of the matrix identity (B2) (see the appendix) we can write equation (4.12) in the form $\dot{L}+[L, \hat{M}]=-3 D^{\prime} L$, where

$$
\hat{M}=M+3 z\left(\left(\beta_{1}-2 c\right) I+B+D\right)=-3 \beta_{2} I-\frac{3}{2}\left(C-D^{\prime}\right)
$$

does not depend on $z$. Using again the identity (Bי

$$
\begin{gathered}
M_{0}=\hat{M}-3(B+D) L=-3 \beta_{2} I-\frac{3}{2}\left(C-D^{\prime}\right)-3(B+D) L \\
=M+3 z\left(\beta_{1}-2 c\right) I+3(B+D)(z I-L)
\end{gathered}
$$

$\left(\beta_{1}, \beta_{2}\right.$ are given in (4.11) $)$. 
The embedding into the Calogero-Moser dynamics discussed above implies that the integrals of motion $I_{k}$ for the dynamical system (4.2) are restrictions of the CalogeroMoser integrals of motion to the subspace of the phase space with $p_{i}=0$. For example:

$$
\begin{aligned}
& I_{2}=\sum_{i<j} \wp\left(x_{i j}\right), \\
& I_{4}=\sum_{i<j<k<l}\left[\wp\left(x_{i j}\right) \wp\left(x_{k l}\right)+\wp\left(x_{i k}\right) \wp\left(x_{j l}\right)+\wp\left(x_{i l}\right) \wp\left(x_{j k}\right)\right] .
\end{aligned}
$$

The spectral curve $\Gamma$ is defined by the equation $R(z, \lambda)=\operatorname{det}(z I-L(\lambda))=0$. It is an $n$-sheet covering of the elliptic curve $\mathcal{E}$ uniformized by the variable $\lambda$ and realized as a factor of the complex plane with respect to the lattice generated by $2 \omega_{1}, 2 \omega_{2}$. Since $L(-\lambda)=-L^{T}(\lambda)$, it is easy to see that the curve $\Gamma$ is equipped with the holomorphic involution $\iota:(z, \lambda) \rightarrow(-z,-\lambda)$. As it was already mentioned, the equation of the spectral curve (the characteristic equation of the Lax matrix) is an integral of motion.

Proposition 4.1 ([20]) For generic values of $x_{i}$ the spectral curve is smooth of genus $g=n$.

\subsection{The wave function as the Baker-Akhiezer function on the spectral curve}

Let $P$ be a point of the spectral curve $\Gamma$, i.e. $P=(z, \lambda)$, where $z$ and $\lambda$ are connected by the equation $R(z, \lambda)=0$. To each point $P$ of the curve there corresponds a single eigenvector $\mathbf{c}(0, P)=\left(c_{1}(0, P), \ldots, c_{n}(0, P)\right)^{T}$ of the matrix $L(t=0, \lambda)$ normalized by the condition $c_{1}(0, P)=1$. The non-normalized components $c_{i}$ are equal to $\Delta_{i}(0, P)$, where $\Delta_{i}(0, P)$ are suitable minors of the matrix $z I-L(0, \lambda)$. They are holomorphic functions on $\Gamma$ outside the points above $\lambda=0$. After normalizing the first component, all other components $c_{i}(0, P)$ become meromorphic functions on $\Gamma$ outside the points $P_{j}$ located above $\lambda=0$. Let $\mathcal{D}^{\prime}$ be the poles divisor of the vector $\mathbf{c}$ with coordinates $c_{i}$. Unlike the spectra curve which is time-independent the divisor $\mathcal{D}^{\prime}$ depends on the initial data.

Lemma 4.1 The sum of the divisors $\mathcal{D}^{\prime}$ and $\iota\left(\mathcal{D}^{\prime}\right)$ is the zero divisor of a holomorphic differential on the spectral curve, i.e. the equation

$$
\mathcal{D}^{\prime}+\iota\left(\mathcal{D}^{\prime}\right)=\mathcal{K}
$$

holds.

Proof. The idea of the proof goes back to the proof of Theorem 4 in [31]. Taking the differential of the eigenvalue equation $(z I-L(\lambda)) \mathbf{c}(P)=0$ and using the equation $\mathbf{c}^{T}(\iota P)(z I-L(\lambda))=0$, which follows from the definition of the involution, we get the equation

$$
\mathbf{c}^{T}(\iota P)(d z I-d L(\lambda)) \mathbf{c}(P)=0,
$$

or

$$
\langle\mathbf{c}(\iota P), \mathbf{c}(P)\rangle d z=\left\langle\mathbf{c}(\iota P), L_{\lambda} \mathbf{c}(P)\right\rangle d \lambda
$$


where $L_{\lambda}=\partial L / \partial \lambda$ and $\langle\mathbf{c}(\iota P), \mathbf{c}(P)\rangle=\sum_{i} c_{i}(\iota P) c_{i}(P)$. For a generic initial data the spectral curve is smooth, i.e. the differentials $d z$ and $d \lambda$ have no common zeros. Then from (4.26) it follows that the zeros of the differential $d \lambda$ (which are ramification points of the covering $\Gamma \rightarrow \mathcal{E})$ coincide with the zeros of the function $\langle\mathbf{c}(\iota P), \mathbf{c}(P)\rangle$. Therefore, the differential

$$
d \Lambda=\frac{d \lambda}{\langle\mathbf{c}(\iota P), \mathbf{c}(P)\rangle}
$$

is a holomorphic differential on the curve $\Gamma$. Its $2 g-2$ zeros at the points, where the vectors $\mathbf{c}(P)$ and $\mathbf{c}(\iota P)$ have poles.

For completeness let us outline the arguments that ultimately lead to the proof of the algebraic integrability of equations (4.2).

A particular case of Theorem 2 in [20] is the following statement.

Theorem 4.3 The function

$$
\hat{\Psi}(x, t, P)=e^{-\zeta(\lambda) x_{1}(0)} \sum_{i=1}^{n} c_{i}(t, P) \Phi\left(x-x_{i}, \lambda\right) e^{z x+z^{3} t}
$$

is the one-point Baker-Akhiezer function on the spectral curve $\Gamma$ with the marked point $P_{\infty}$ (one of pre-images of $\lambda=0$ ) corresponding to the divisor $\mathcal{D}=\mathcal{D}^{\prime}+P_{\infty}$.

By definition the function $\hat{\Psi}$ has poles at $x_{i}(t)$. From the theta-functional formula (3.33) for the Baker-Akhiezer function it follows that $x_{i}$ are zeros of the second factor in the denominator, i.e. they are roots in $x$ of the equation

$$
\theta\left(\vec{U}_{1} x+\vec{U}_{3} t-\vec{A}(\mathcal{D})-\vec{K}+\vec{A}\left(P_{\infty}\right)\right)=0 .
$$

From Lemma4.1 it follows that the pole divisor $\mathcal{D}$ of the Baker-Akhiezer function satisfies the equation

$$
\mathcal{D}+\iota \mathcal{D}-2 P_{\infty}=\mathcal{K}
$$

where $\mathcal{K}$ is the canonical class. This is precisely the condition (3.50) on the divisor of poles of the Baker-Akhiezer function for algebraic-geometric solutions to the CKP equation. This completes the proof of (4.5) since (4.29) is equivalent to equation (3.55) for the vector $\vec{Z}$ in (4.5).

\subsection{Degenerations of elliptic solutions}

\subsubsection{Trigonometric solutions}

In the degenerate case, when one of the periods tends to infinity, the elliptic solutions become trigonometric (hyperbolic). We consider trigonometric solutions which vanish at infinity:

$$
u(x, \mathbf{t})=-\frac{1}{2} \sum_{i=1}^{n} \frac{\gamma^{2}}{\sinh ^{2}\left(\gamma\left(x-x_{i}(\mathbf{t})\right)\right.},
$$


where $\gamma$ is a complex parameter. When $\gamma$ is purely imaginary (respectively, real), one deals with trigonometric (respectively, hyperbolic) solutions. The equations of motion for the poles are

$$
\dot{x}_{i}=3 \sum_{k \neq i} \frac{\gamma^{2}}{\sinh ^{2}\left(\gamma\left(x_{i}-x_{k}\right)\right)}-\gamma^{2} .
$$

Tending the spectral parameter $\lambda$ to infinity, we find the Lax matrix in the form

$$
L_{i j}=-\frac{\gamma\left(1-\delta_{i j}\right)}{\sinh \left(\gamma\left(x_{i}-x_{j}\right)\right)}
$$

Note that it is antisymmetric.

As is shown in [32], the KP tau-function for trigonometric solutions has the following determinant representation:

$$
\tau^{\mathrm{KP}}(x, \mathbf{t})=\operatorname{det}_{n \times n}\left(e^{2 \gamma x} I-\exp \left(-\sum_{k \geq 1} t_{k} \mathcal{L}_{k}\right) e^{2 \gamma X_{0}}\right)=\prod_{j=1}^{n}\left(e^{2 \gamma x}-e^{2 \gamma x_{j}(\mathbf{t})}\right),
$$

where $X_{0}=\operatorname{diag}\left(x_{1}(0), \ldots, x_{n}(0)\right)$ and

$$
\mathcal{L}_{k}=\left(L_{0}+\gamma I\right)^{k}-\left(L_{0}-\gamma I\right)^{k}, \quad L_{0}=L(\mathbf{t}=0) .
$$

We see that $\mathcal{L}_{k}$ is a polynomial in $L_{0}$ of degree $k-1$. If $k$ is even (respectively, odd), $\mathcal{L}_{k}$ contains only odd (respectively, even) powers of $L_{0}$.

It is easy to see that this tau-function satisfies the conditions (2.48), and, therefore, gives rise to the CKP tau-function

$$
\tau\left(x, \mathbf{t}_{\mathrm{o}}\right)=\left(\operatorname{det}_{n \times n}\left(e^{2 \gamma x} I-\exp \left(-\sum_{k \geq 1, k \text { odd }} t_{k} \mathcal{L}_{k}\right) e^{2 \gamma X_{0}}\right)\right)^{1 / 2} .
$$

Indeed, we have

$$
\begin{aligned}
& \left.\partial_{t_{2 m}} \log \tau^{\mathrm{KP}}\right|_{t_{2 k}=0}=\left.\partial_{t_{2 m}} \operatorname{tr} \log \left(e^{2 \gamma x} I-\exp \left(-\sum_{k \geq 1} t_{k} \mathcal{L}_{k}\right) e^{2 \gamma X_{0}}\right)\right|_{t_{\mathrm{e}}=0} \\
= & \operatorname{tr}\left[\mathcal{L}_{2 m} \exp \left(-\sum_{k \geq 1, k \text { odd }} t_{k} \mathcal{L}_{k}\right)\left(e^{2 \gamma x} I-\exp \left(-\sum_{k \geq 1, k \text { odd }} t_{k} \mathcal{L}_{k}\right) e^{2 \gamma X_{0}}\right)^{-1}\right] .
\end{aligned}
$$

But this is zero for all $m \geq 1$ because $\operatorname{tr} L_{0}^{2 l-1}=0$ for all $l \geq 1$ and, as it was said above, $\mathcal{L}_{2 m}$ contains only odd powers of $L_{0}$ while all other $\mathcal{L}_{k}$ in this expression contain only even powers of $L_{0}$.

\subsubsection{Rational solutions}

In the most degenerate case, when both periods tend to infinity, $\wp(x) \rightarrow 1 / x^{2}$ and the elliptic solutions become rational:

$$
u(x, \mathbf{t})=-\frac{1}{2} \sum_{i=1}^{n} \frac{1}{\left(x-x_{i}(\mathbf{t})\right)^{2}} .
$$


This corresponds to the limit $\gamma \rightarrow 0$ in the trigonometric solutions. The equations of motion for the poles are

$$
\dot{x}_{i}=3 \sum_{k \neq i} \frac{1}{\left(x_{i}-x_{k}\right)^{2}} .
$$

Tending the spectral parameter $\lambda$ to infinity, $\lambda=\infty$, we find the (antisymmetric) Lax matrix in the form

$$
L_{i j}=-\frac{1-\delta_{i j}}{x_{i}-x_{j}}
$$

It is known that the KP tau-function for rational solutions has the following determinant representation (see, e.g. [33]):

$$
\tau^{\mathrm{KP}}(x, \mathbf{t})=\operatorname{det}_{n \times n}\left(x I-X_{0}+\sum_{k \geq 1} k t_{k} L_{0}^{k-1}\right)=\prod_{j=1}^{n}\left(x-x_{j}(\mathbf{t})\right),
$$

where $X_{0}=\operatorname{diag}\left(x_{1}(0), \ldots, x_{n}(0)\right)$ and $L_{0}=L(\mathbf{t}=0)$. It is easy to see that this taufunction satisfies the conditions (2.48), and, therefore, gives rise to the CKP tau-function

$$
\tau\left(x, \mathbf{t}_{\mathrm{o}}\right)=\left(\operatorname{det}_{n \times n}\left(x I-X_{0}+\sum_{k \geq 1, k \text { odd }} k t_{k} L_{0}^{k-1}\right)\right)^{1 / 2}
$$

Indeed, we have

$$
\begin{aligned}
& \left.\partial_{t_{2 m}} \log \tau^{\mathrm{KP}}\right|_{t_{2 k}=0}=\left.\partial_{t_{2 m}} \operatorname{tr} \log \left(x I-X_{0}+\sum_{k \geq 1} k t_{k} L_{0}^{k-1}\right)\right|_{t_{\mathrm{e}}=0} \\
= & 2 m \operatorname{tr}\left[L_{0}^{2 m-1}\left(\left(x+t_{1}\right) I-X_{0}+3 t_{3} L_{0}^{2}+5 t_{5} L_{0}^{4}+\ldots\right)^{-1}\right]=0
\end{aligned}
$$

for all $m \geq 1$ because $\operatorname{tr} L_{0}^{2 l-1}=0$ for all $l \geq 1$.

\section{Concluding remarks}

The main result of this paper is the identification of the CKP hierarchy as the hierarchy of odd times flows of the KP hierarchy restricted onto the locus of its turning points. It suggests that a similar result might be valid for the BKP hierarchy. Namely, we conjecture that the BKP hierarchy can be identified with the restriction of odd times flows of the KP hierarchy onto the locus which in the Sato formulation is defined by the equation

$$
\left(\mathcal{L}^{3}\right)_{+}=\partial_{x}^{3}+6 u \partial_{x}
$$

i.e. the coefficient at the zero power of $\partial_{x}$ in $\mathcal{L}^{3}$ vanishes. In terms of the tau-function, this condition means that

$$
\left.\left(\partial_{t_{2}}+\partial_{t_{1}}^{2}\right) \log \tau^{\mathrm{KP}}\right|_{\mathbf{t}_{\mathrm{e}}}=0
$$

for all $t_{1}, t_{3}, t_{5}, \ldots$. The latter is an analog of equation (2.48) defining turning points of the KP hierarchy.

Another interesting problem we plan to consider in the future is the Hamiltonian theory of equations of motion for poles of elliptic solutions to the CKP hierarchy. In 
section 4 we have derived these equation in two ways. First, these equations can be obtained by restricting the higher equations of motion of the elliptic Calogero-Moser system onto the locus of its turning points. As a corollary of this, we have presented solutions of these equations in the implicit function form using theta-function of the spectral curve. The second approach to the equations of motion is via the "generating linear problem" scheme which allows us to define the corresponding spectral curve and to prove that it is time-independent in a direct way (i.e. without any reference to the elliptic Calogero-Moser system).

As it was shown earlier in [24], the phase space of the elliptic CKP system can be identified with the total space of the Prym varieties bundle over the space of the spectral curves. Under this identification the equations of motion become linear on the fibers. Such picture is characteristic for algebraically integrable Hamiltonian systems. However, the authors's attempts to find the corresponding Hamiltonian formulation of equations (4.2) by a direct guess or by more advanced machinery proposed in [2, 21, 34] have failed so far.

\section{Acknowledgments}

We thank S. Natanzon for discussions. The research has been funded within the framework of the HSE University Basic Research Program and the Russian Academic Excellence Project '5-100'.

\section{Appendix A: Proof of Lemma 2.1}

In this appendix we give a sketch of proof of Lemma 2.1, i.e. we are going to prove that the conditions (2.53) and

$$
\left.\partial_{x} \partial_{t_{4}} \log \tau^{\mathrm{KP}}\right|_{\mathbf{t}_{\mathrm{e}}=0}=\left.\partial_{x} \partial_{t_{6}} \log \tau^{\mathrm{KP}}\right|_{\mathbf{t}_{\mathrm{e}}=0}=\ldots=0 .
$$

follow from the constraint

$$
\left.\partial_{t_{2}} \log \tau^{\mathrm{KP}}\right|_{\mathbf{t}_{\mathrm{e}}=0}=0 \quad \text { for all } t_{1}, t_{3}, t_{5}, \ldots
$$

(see (2.48) ) provided $\tau^{\mathrm{KP}}$ is a KP tau-function, i.e. satisfies all the equations of the KP hierarchy.

We use the representation of the KP hierarchy in the unfolded form suggested in [35, 36], see also section 3.2 of [37]. Set $F=\log \tau^{\mathrm{KP}}$ and $F_{k_{1}, \ldots, k_{m}}=\partial_{t_{k_{1}}} \ldots \partial_{t_{k_{m}}} F$. Then the KP hierarchy can be written in the form

$$
F_{k_{1}, \ldots, k_{m}}=\sum_{n \geq 1} \sum R_{k_{1}, \ldots, k_{m}}^{(n)}\left(\begin{array}{ccc}
s_{1} & \ldots & s_{n} \\
r_{1} & \ldots & r_{n}
\end{array}\right) \partial_{x}^{r_{1}} F_{s_{1}} \ldots \partial_{x}^{r_{n}} F_{s_{n}}
$$

where $m \geq 2$ and $R_{k_{1}, \ldots, k_{m}}^{(n)}\left(\begin{array}{ccc}s_{1} & \ldots & s_{n} \\ r_{1} & \ldots & r_{n}\end{array}\right)$ are universal rational coefficients. The second 
sum is taken over all matrices $\left(\begin{array}{lll}s_{1} & \ldots & s_{n} \\ r_{1} & \ldots & r_{n}\end{array}\right)$ such that $s_{i}, r_{i} \geq 1$ with the conditions

$$
\sum_{i=1}^{n}\left(s_{i}+r_{i}\right)=\sum_{i=1}^{m} k_{i}, \quad \sum_{i=1}^{n} r_{i} \geq n+m-2
$$

For example [35],

$$
F_{2,3}=\frac{3}{2} \partial_{x} F_{4}-\frac{3}{2} \partial_{x}^{3} F_{2}-3 \partial_{x} F_{2} \partial_{x}^{2} F
$$

From the fact that if $\tau^{\mathrm{KP}}(x, \mathbf{t})$ is a tau-function, then $\tau^{\mathrm{KP}}(-x,-\mathbf{t})$ is a tau-function, too (this is a corollary of the Hirota equations), it follows that

$$
\text { if } \sum_{i=1}^{n}\left(r_{i}-1\right)-m \equiv 1(\bmod 2) \text {, then } R_{k_{1}, \ldots, k_{m}}^{(n)}\left(\begin{array}{ccc}
s_{1} & \ldots & s_{n} \\
r_{1} & \ldots & r_{n}
\end{array}\right)=0 \text {. }
$$

First we prove (A1). The proof is by induction. We assume that (A1) is true for $\partial_{x} F_{2}, \ldots, \partial_{x} F_{2 k}$ (this is certainly true if $k=1$ ) and will deduce from (A3) that it is true for $k \rightarrow k+1$. From (A2) and (A3) at $m=2$ we have:

$$
\begin{gathered}
0=F_{2,2 k+1}=\sum_{s_{1}+r_{1}=2 k+3} R_{2,2 k+1}^{(1)}\left(\begin{array}{c}
s_{1} \\
r_{1}
\end{array}\right) \partial_{x}^{r_{1}} F_{s_{1}} \\
+\sum_{s_{1}+s_{2}+r_{1}+r_{2}=2 k+3} R_{2,2 k+1}^{(2)}\left(\begin{array}{cc}
s_{1} & s_{2} \\
r_{1} & r_{2}
\end{array}\right) \partial_{x}^{r_{1}} F_{s_{1}} \partial_{x}^{r_{2}} F_{s_{2}}+\ldots
\end{gathered}
$$

Separating the term with $r_{1}=1$ in the first sum in the right hand side of (A7), we write it as

$$
0=F_{2,2 k+1}=R_{2,2 k+1}^{(1)}\left(\begin{array}{c}
2 k+2 \\
1
\end{array}\right) \partial_{x} F_{2 k+2}+\text { all the rest. }
$$

Now, recalling the condition (A6), we see that the non-zero coefficients at the different terms in the right hand side are when $\sum_{i=1}^{n} s_{i}=n-1(\bmod 2)$. From this it follows that for both odd and even $n$ at least one of the $s_{i}$ 's must be even (and less then $2 k+2$ ). Therefore, "all the rest" terms vanish by the induction assumption. Since the coefficient $R_{2,2 k+1}^{(1)}\left(\begin{array}{c}2 k+2 \\ 1\end{array}\right)$ is not equal to zero (see [35]), we conclude from (쇼) that $\partial_{x} F_{2 k+2}=$ 0 .

Next we are going to prove that if $\partial_{x} F_{2 k}=0$ for all $k \geq 1$ and all $t_{1}, t_{3}, \ldots$, then $F_{k_{1}, \ldots, k_{m}}=0$ for all even $k_{1}, \ldots, k_{m}$ and odd $m \geq 3$. As soon as $m+1$ and all $k_{i}$ 's are even, we can, using (A4), rewrite the condition (A6) in the form

$$
\sum_{i=1}^{n} s_{i} \equiv n(\bmod 2)
$$

But if at least one of $s_{i}$ in (A3) is even, then the corresponding term vanishes because $F_{2 k}=0$ for all $k \geq 1$. Therefore, all the $s_{i}$ 's must be odd, i.e., $s_{i}=2 l_{i}+1$ and so the condition (A9) is satisfied which means that the coefficient $R_{k_{1}, \ldots, k_{m}}^{(n)}\left(\begin{array}{lll}s_{1} & \ldots & s_{n} \\ r_{1} & \ldots & r_{n}\end{array}\right)$ vanishes. This proves that $F_{k_{1}, \ldots, k_{m}}=0$. 


\section{Appendix B: Proof of equation (4.21)}

Here we prove the matrix identity (4.21).

First of all we note that $\dot{L}_{i k}=-\left(\dot{x}_{i}-\dot{x}_{k}\right) \Phi^{\prime}\left(x_{i}-x_{k}\right)$, and, therefore, we have $\dot{L}=$ $-[\dot{X}, B]$. To transform the commutators $[L, B]+[L, D]$, we use the identity

$$
\Phi(x) \Phi^{\prime}(y)-\Phi(y) \Phi^{\prime}(x)=\Phi(x+y)(\wp(x)-\wp(y)) .
$$

With the help of it we get for $i \neq k$

$$
\begin{gathered}
-([L, B]+[L, D])_{i k} \\
=\sum_{j \neq i, k} \Phi\left(x_{i}-x_{j}\right) \Phi^{\prime}\left(x_{j}-x_{k}\right)-\sum_{j \neq i, k} \Phi^{\prime}\left(x_{i}-x_{j}\right) \Phi\left(x_{j}-x_{k}\right) \\
\quad+\Phi\left(x_{i}-x_{k}\right)\left(\sum_{j \neq k} \wp\left(x_{j}-x_{k}\right)-\sum_{j \neq i} \wp\left(x_{i}-x_{j}\right)\right)=0,
\end{gathered}
$$

so we see that $[L, B]+[L, D]$ is a diagonal matrix. To find its matrix elements, we use the limit of (B1) at $y=-x$ :

$$
\Phi(x) \Phi^{\prime}(-x)-\Phi(-x) \Phi^{\prime}(x)=\wp^{\prime}(x)
$$

which leads to

$$
\begin{gathered}
-([L, B]+[L, D])_{i i} \\
=\sum_{j \neq i}\left(\Phi\left(x_{i}-x_{j}\right) \Phi^{\prime}\left(x_{j}-x_{i}\right)-\Phi^{\prime}\left(x_{i}-x_{j}\right) \Phi\left(x_{j}-x_{i}\right)\right)=\sum_{j \neq i} \wp^{\prime}\left(x_{i}-x_{j}\right)=D_{i i}^{\prime},
\end{gathered}
$$

so we finally obtain the matrix identity

$$
[L, B]+[L, D]=-D^{\prime}
$$

Combining the derivatives of (B1) w.r.t. $x$ and $y$, we obtain the identity

$$
\Phi(x) \Phi^{\prime \prime}(y)-\Phi(y) \Phi^{\prime \prime}(x)=2 \Phi^{\prime}(x+y)(\wp(x)-\wp(y))+\Phi(x+y)\left(\wp^{\prime}(x)-\wp^{\prime}(y)\right)
$$

which allows us to prove the matrix identity

$$
[L, C]=-2[D, B]+D^{\prime} L+L D^{\prime},
$$

which is used, together with $(\overline{\mathrm{B} 2})$, to transform $\dot{L}+[L, M]$ to the form (4.21).

\section{References}

[1] V.E. Zakharov and A.B. Shabat, A scheme for integrating the nonlinear equations of mathematical physics by the method of the inverse scattering problem, I, Funk. Anal. i ego Pril. 8:3 (1974) 43--53.

[2] I. Krichever and D.H. Phong, On the integrable geometry of soliton equations and $N=2$ supersymmetric gauge theories, J. Diff. Geom. 45:2 (1997) 349-389. 
[3] M. Sato, Soliton equations and the universal Grassmann manifold, Math. Notes. Series 18 (1984), Sophia University, Tokyo.

[4] T. Takebe, From general Zakharov-Shabat equations to the KP and the Toda lattice hierarchies, Int. J. Mod. Phys. A 7 (1992) No. supp01b, 923-939.

[5] M. Jimbo and T. Miwa, Soliton equations and infinite dimensional Lie algebras, Publ. RIMS, Kyoto University 19 (1983) 943-1001.

[6] E. Date, M. Jimbo, M. Kashiwara and T. Miwa, Transformation groups for soliton equations, in "Nonlinear Integrable Systems - Classical Theory and Quantum theory", M. Jimbo and T. Miwa (eds.), World Sci., Singapore, 1983, pp. 39-119.

[7] E. Date, M. Jimbo, M. Kashiwara and T. Miwa, KP hierarchy of orthogonal and symplectic type - Transformation groups for soliton equations VI, J. Phys. Soc. Japan 50 (1981) 3813-3818.

[8] A. Dimakis and F. Müller-Hoissen, BKP and CKP revisited: the odd KP system, Inverse Problems 25 (2009) 045001, arXiv:0810.0757.

[9] L. Chang and C.-Z. Wu, Tau function of the CKP hierarchy and non-linearizable Virasoro symmetries, Nonlinearity 26 (2013) 2577-2596.

[10] J. Cheng and J. He, The "ghost" symmetry in the CKP hierarchy, Journal of Geometry and Physics, 80 (2014) 49-57.

[11] J. van de Leur, A. Orlov and T. Shiota, CKP hierarchy, bosonic tau function and bosonization formulae, SIGMA 8 (2012) 036, arXiv:1102.0087

[12] I. Krichever, Integration of nonlinear equations by methods of algebraic geometry, Funkt. Anal. i ego Pril. 11 (1977) 15-31.

[13] I. Krichever, Methods of algebraic geometry in the theory of non-linear equations, Russian Math. Surveys 32 (1977) 185-213.

[14] H. Airault, H.P. McKean, and J. Moser, Rational and elliptic solutions of the Korteweg-De Vries equation and a related many-body problem, Commun. Pure Appl. Math., 30 (1977) 95-148.

[15] F. Calogero, Solution of the one-dimensional $N$-body problems with quadratic and/or inversely quadratic pair potentials, J. Math. Phys. 12 (1971) 419-436.

[16] F. Calogero, Exactly solvable one-dimensional many-body systems, Lett. Nuovo Cimento 13 (1975) 411-415.

[17] J. Moser, Three integrable Hamiltonian systems connected with isospectral deformations, Adv. Math. 16 (1975) 197-220.

[18] I.M. Krichever, Rational solutions of the Kadomtsev-Petviashvili equation and integrable systems of $N$ particles on a line, Funct. Anal. Appl. 12:1 (1978) 59-61.

[19] D.V. Chudnovsky, G.V. Chudnovsky, Pole expansions of non-linear partial differential equations, Nuovo Cimento 40B (1977) 339-350. 
[20] I.M. Krichever, Elliptic solutions of the Kadomtsev-Petviashvili equation and integrable systems of particles, Funk. Anal. i Ego Pril. 14:4 (1980) 45-54 (in Russian); English translation: Functional Analysis and Its Applications 14:4 (1980) 282 - 290.

[21] I. Krichever, Elliptic solutions to difference non-linear equations and nested Bethe ansatz equations, CRM Series in Mathematical Physics, Calogero-Moser-Sutherland Models, pp. 249-271, J.F. van Diejen, L. Vinet (eds.), arXiv:solv-int/9804016.

[22] D. Rudneva and A. Zabrodin, Dynamics of poles of elliptic solutions to BKP equation, Journal of Physics A: Math. Theor. 53 (2020) 075202.

[23] A. Zabrodin, Elliptic solutions to integrable nonlinear equations and many-body systems, Journal of Geometry and Physics 146 (2019) 103506.

[24] I. Krichever, N. Nekrasov, Towards Lefschets thimbles in sigma models, II, to appear

[25] J.D. Fay, Theta functions on Riemann surfaces, Lect. Notes Math., Vol. 352, Berlin, Heidelberg, New York: Springer, 1973.

[26] D. Mumford, Tata Lectures on Theta I, II, Progress in Mathematics, Vol. 28, 43, Birkhäuser, Boston, Basel, Stuttgart, 1983, 1984.

[27] I. Cherednik, Differential equations for the Baker-Akhiezer functions of algebraic curves, Funct. Anal. and Appl. 12 (1978) 45-52.

[28] I. Krichever, T. Shiota, Soliton equations and the Riemann-Schottky problem, Handbook of moduli, Vol. II, 205-258, Adv. Lect. Math. (ALM), 25, Int. Press, Somerville, MA, 2013.

[29] B. Dubrovin, Theta-functions and nonlinear equations, Uspekhi Mat. Nauk 36 (1981) 12-72.

[30] S. Manakov, Method of inverse scattering problem and two-dimensional evolution equations, Uspekhi Mat. Nauk 31 (1976) 245-246.

[31] I. Krichever, The spectral theory of "finite-gap" non-stationary Schrödinger operators. The non-stationary Peierls model, Funk. Anal. i ego Pril. 20:3 (1986) 42-54.

[32] A. Zabrodin, KP hierarchy and trigonometric Calogero-Moser hierarchy, Journal of Mathematical Physics 61 (2020) 043502.

[33] T. Shiota, Calogero-Moser hierarchy and KP hierarchy, J. Math. Phys. 35 (1994) $5844-5849$.

[34] I. Krichever and D.H. Phong, Symplectic forms in the theory of solitons, Surveys in Differential Geometry IV (1998), edited by C.L. Terng and K. Uhlenbeck, 239-313, International Press, arXiv:hep-th/9708170.

[35] S. Natanzon, Formulas for $A_{n}$ and $B_{n}$-solutions of WDVV equations, J. Geom. Phys. 39 (2001) 323-336. 
[36] S. Natanzon, Witten solution for the Gelfand-Dikii hierarchy, Funk. Anal. i ego Prilozh. 37:1 (2003) 25-37 (English translation: Func. Anal. Appl. 37:1 (2003) 2131).

[37] S. Natanzon and A. Zabrodin, Formal solution to the KP hierarchy, J. Phys. A: Math. Theor. 49 (2016) 145206. 